\title{
COMMON VALUES, UNOBSERVED HETEROGENEITY, AND ENDOGENOUS ENTRY IN U.S. OFFSHORE OIL LEASE AUCTION
}

\author{
Giovanni Compiani \\ Philip Haile \\ Marcelo Sant'Anna \\ Working Paper 24795 \\ http://www.nber.org/papers/w24795 \\ NATIONAL BUREAU OF ECONOMIC RESEARCH \\ 1050 Massachusetts Avenue \\ Cambridge, MA 02138 \\ July 2018
}

We are grateful to Ken Hendricks and Rob Porter, who generously provided the data, answered our many questions, and gave valuable feedback on early drafts. We also benefited from the comments of Estelle Cantillon, Xiaohong Chen, and numerous seminar and conference participants. The views expressed herein are those of the authors and do not necessarily reflect the views of the National Bureau of Economic Research.

NBER working papers are circulated for discussion and comment purposes. They have not been peer-reviewed or been subject to the review by the NBER Board of Directors that accompanies official NBER publications.

(C) 2018 by Giovanni Compiani, Philip Haile, and Marcelo Sant'Anna. All rights reserved. Short sections of text, not to exceed two paragraphs, may be quoted without explicit permission provided that full credit, including $(\odot$ notice, is given to the source. 
Common Values, Unobserved Heterogeneity, and Endogenous Entry in U.S. Offshore Oil

Lease Auction

Giovanni Compiani, Philip Haile, and Marcelo Sant'Anna

NBER Working Paper No. 24795

July 2018

JEL No. L0

\begin{abstract}
An oil lease auction is the classic example motivating a common values model. However, formal testing for common values has been hindered by unobserved auction-level heterogeneity, which is likely to affect both participation in an auction and bidders' willingness to pay. We develop and apply an empirical approach for first-price sealed bid auctions with affiliated values, unobserved heterogeneity, and endogenous bidder entry. The approach also accommodates spatial dependence and sample selection. Following Haile, Hong and Shum (2003), we specify a reduced form for bidder entry outcomes and rely on an instrument for entry. However, we relax their control function requirements and demonstrate that our specification is generated by a fully specified game motivated by our application. We show that important features of the model are nonparametrically identified and propose a semiparametric estimation approach designed to scale well to the moderate sample sizes typically encountered in practice. Our empirical results show that common values, affiliated private information, and unobserved heterogeneity-three distinct phenomena with different implications for policy and empirical work-are all present and important in U.S. offshore oil and gas lease auctions. We find that ignoring unobserved heterogeneity in the empirical model obscures the presence of common values. We also examine the interaction between affiliation, the winner's curse, and the number of bidders in determining the aggressiveness of bidding and seller revenue
\end{abstract}

Giovanni Compiani

University of California at Berkeley

2220 Piedmont Ave

Berkeley, CA 94720

gcompiani@berkeley.edu

Philip Haile

Department of Economics

Yale University

37 Hillhouse Avenue

P.O. Box 208264

New Haven, CT 06520

and NBER

philip.haile@yale.edu
Marcelo Sant'Anna

FGV EPGE

Praia de Botafogo, 190/1100

Rio de Janeiro, RJ, 22250-900

Brazil

msantanna@gmail.com 


\section{Introduction}

In many auction settings it is natural to presume that important information commonly known among bidders is unobserved by the econometrician. Ignoring such unobserved heterogeneity can lead to a variety of errors. One may infer too much within-auction correlation in bidders' private information, as well as too much crossauction variation in this information, leading to incorrect conclusions about such issues as bidder market power, the division of surplus, and optimal auction design.$^{1}$ In a first-price auction, unobserved heterogeneity presents a particular challenge because standard identification approaches exploit the insight that bidders' equilibrium beliefs about the competition can be inferred from observed distributions of rivals' bids.2 With unobserved auction-level heterogeneity, bidders' beliefs condition on information unavailable to the econometrician. A further problem is that auction-level unobservables are likely to affect not only bids but also bidder participation. Such endogenous bidder entry threatens several identification and testing approaches relying on exogenous variation in the level of competition $!^{3}$

We propose an empirical model of entry and bidding in first-price auctions with affiliated values and unobserved heterogeneity. We show nonparametric identification of key features and propose a semiparametric estimation approach. We apply the approach to auctions of offshore oil and gas leases in the United States Outer Continental Shelf ("OCS") to evaluate the importance of unobserved heterogeneity, test the hypothesis of equilibrium bidding, assess the effect of competition on bidding and revenues, and test for common values. The last of these is of particular interest.

\footnotetext{
${ }^{1}$ See, e.g., Krasnokutskaya (2011), Krasnokutskaya and Seim (2011), Athey, Levin, and Seira (2011), and Roberts (2013).

${ }^{2}$ See, e.g., Laffont and Vuong (1993), Guerre, Perrigne, and Vuong (2000), Li, Perrigne, and Vuong (2002), Hendricks, Pinkse, and Porter (2003), and Athey and Haile (2006, 2007).

${ }^{3}$ See, e.g., Gilley and Karels (1981), Athey and Haile (2002), Haile, Hong, and Shum (2003), Guerre, Perrigne, and Vuong (2009), Campo, Guerre, Perrigne, and Vuong (2011), and Gillen (2010).
} 
Common values can have important implications in the auction context and beyond (e.g., Akerlof (1970) ), and an auction's clear rules offer hope of confronting our intuition about latent information structures with formal hypothesis testing in a setting where the model can be tightly linked to the actual market institution. Although an auction of drilling rights is often cited as an example of a common values setting, formal testing for common values has been hindered by the confounding effects of unobserved heterogeneity. Indeed, we reject private values in favor of common values only when accounting for unobserved heterogeneity and endogenous entry. More broadly, we find that affiliated private information, common values, and common knowledge unobservables - three distinct phenomena with different implications for policy and empirical work 4 are all present and important in OCS auctions.

Our empirical study is perhaps most closely related to that of Hendricks, Pinkse, and Porter (2003) ("HPP"), who focused on testable implications of a pure common values model $5^{5}$ Our work is complementary to that of HPP. We allow the pure common values model but do not assume it, and we neither exploit nor rely on estimates of realized tract values. HPP point out that tests for common values would be difficult to apply due to likely correlation between bidder entry and auction-level unobservables.$^{6}$ Our study also complements the simultaneous work of Aradillas-Lopez, Haile, Hendricks, and Porter (2017), which evaluates implications of competitive bidding in OCS auctions following the introduction of "area-wide leasing" in 1983. Here we consider only the period 1954-1983, when the evidence in Haile, Hendricks, and Porter

\footnotetext{
${ }^{4}$ For example, affiliation leads to the "linkage principle" (Milgrom and Weber (1982), Milgrom (1987)) whereas common values leads to the "winner's curse," each with important implications for auction design. Unobserved heterogeneity, which is held fixed in auction theory, implies neither affiliation nor common values but creates challenges for identification.

${ }^{5}$ Earlier work on OCS auctions includes Gilley and Karels (1981), Hendricks, Porter, and Boudreau (1987), Hendricks and Porter (1988), and Hendricks, Porter, and Spady (1989).

${ }^{6} \mathrm{HPP}$ partially control for unobserved heterogeneity by conditioning on two categories of tractsthose with high or low numbers of potential bidders, measured as the number of large firms ever to bid on a tract within a certain distance of the tract offered.
} 
(2010) and Aradillas-Lopez et al. supports the assumption of competitive bidding.

Prior work on testing for common values in auctions includes Paarsch (1992), Athey and Haile (2002), Bajari and Hortaçsu (2003), HHS, Hill and Schneyerov (2014), and Hortaçsu and Kastl (2012). Most of this work, like ours, exploits the fact that in a common values auction the winner's curse becomes more severe as the number of competitors grows (all else equal). Our approach to identification and testing is most similar to that in HHS, who studied timber auctions. We drop their assumptions of discrete unobserved heterogeneity and entry outcomes that are strictly increasing in the unobservable. This makes the identification problem substantially more challenging and requires a different estimation strategy.

We are not the first to consider first-price auctions with unobserved heterogeneity. Haile and Kitamura (2018) review existing econometric approaches. All require compromises. Several (e.g., Krasnokutskaya (2011), Hu, McAdams, and Shum (2013)) require that bidders have independent types, so that all correlation among bids can be attributed to unobserved heterogeneity.7 Other approaches use a control function strategy requiring strict monotonicity between an observed outcome and the unobserved heterogeneity (e.g., Campo, Perrigne, and Vuong (2003), Haile, Hong, and Shum (2003) ("HHS"), Guerre, Perrigne, and Vuong (2009), Roberts (2013)). Simultaneous work by Kitamura and Laage (2017) proposes an approach allowing affiliated types but requiring that the unobservable be discrete (cf. HHS and Hu, McAdams, and Shum (2013)) and enter through a separable structure similar to that in Krasnokutskaya (2011). Finally, while control function approaches can provide a strategy for isolating exogenous variation in bidder entry, others generally do not.

Our approach requires compromises as well. We rely on an index assumption similar to that in Krasnokutskaya (2011) and Kitamura and Laage (2017) and spec-

\footnotetext{
${ }^{7}$ See also the partial identification results in Armstrong (2013). An exception among approaches building on the measurement error literature is Balat (2011). His extension of $\mathrm{Hu}$, McAdams, and Shum (2013) exploits observation of potential bidders' entry decisions at two sequential stages.
} 
ify only a reduced form for bidder entry. The latter rules out selective entry ${ }^{8}$ and cannot be used to evaluate interventions that would alter the map between auction characteristics and entry outcomes $9^{9}$ And like HHS, we require an instrument for entry. But our approach also offers advantages that are attractive for our application. It avoids the requirement of independent bidder types and provides a strategy for exploiting exogenous sources of variation in bidder entry. This combination of features is particularly important in our application. Common values settings generally demand that we allow correlated types (signals), and our test for common values exploits exogenous variation in entry arising through an instrumental variable. We also avoid the strict monotonicity requirement of the control function approach and show that our empirical model can be derived from a two-stage game motivated by our application - an entry stage à la Berry (1992) in which bidders choose whether to acquire a signal of the good's value, followed by competitive bidding à la Milgrom and Weber (1982). In this example, unobserved heterogeneity may have arbitrary dimension; may exhibit spatial dependence and correlation with observables; and may affect sample selection. Finally, although we require an instrument satisfying certain conditions for point identification, our formal analysis also isolates the extent to which one would rely on parametric structure for identification if in practice one has instruments generating only limited (even no) variation in entry.

The following section describes our model. In Section 3 we address nonparametric identification. Section 4 describes our proposed estimation method. We then narrow our focus to the OCS oil lease auctions, with baseline estimates presented in section 5. We present the tests for common values in section 6, with several alternative specifications examined in section 7. We conclude in section 8 .

${ }^{8}$ cf. Marmer, Schneyerov, and Xu (2013), Gentry and Li (2014), Bhattacharya, Roberts, and Sweeting (2014), or Kong (2017a).

${ }^{9}$ However, once the bidding model is identified it may become possible to identify a given structural model of entry consistent with the reduced form, allowing evaluation of such counterfactuals. 


\section{Model}

We consider a standard model of first-price sealed bid auctions with symmetric affiliated values, extended to allow for auction-level heterogeneity and endogenous bidder entry. Each auction $t$ is associated with observed characteristics $X_{t} \in \mathbb{X}$ and a scalar unobservable $U_{t}$. Without further loss, we let $U_{t}$ be uniformly distributed on $[0,1]$. We also assume independence between $X_{t}$ and $U_{t}$.

Assumption 1. $X_{t} \Perp U_{t}$.

The restriction to a scalar unobservable independent of $X_{t}$ is less restrictive than it appears. We show below that this representation can be derived-without loss of generality for most purposes motivating estimation of an auction model - from a model in which auction-level unobservables have arbitrary dimension and arbitrary dependence with $X_{t}$. In that model, the weak monotonicity conditions required below are also obtained as results rather than assumptions.

For each auction $t$ we postulate a two-stage process in which entry is followed by bidding. We do not specify a particular model of entry; rather, we posit a reduced form for the entry outcome and assume Bayes Nash equilibrium in the auction stage. The number of bidders entering auction $t$ is denoted by $N_{t}$. Bidders are assumed risk neutral. Bidder $i$ 's valuation for the good offered is denoted by $V_{i t}$. Upon entering, $i$ observes a private signal $S_{i t} \in[\underline{s}, \bar{s}]$ of $V_{i t}$. Let $V_{t}=\left(V_{1 t}, \ldots, V_{N_{t} t}\right)$, $S_{t}=\left(S_{1 t}, \ldots, S_{N_{t} t}\right)$, and $S_{-i t}=S_{t} \backslash S_{i t}$.

The bidding stage follows Milgrom and Weber (1982). The realizations of $\left(N_{t}, X_{t}, U_{t}\right)$ are common knowledge among bidders, as is the distribution of $\left(S_{t}, V_{t}\right) \mid\left(N_{t}, X_{t}, U_{t}\right){ }^{10}$

\footnotetext{
${ }^{10}$ Although standard, the assumption that bidders observe $N_{t}$ may be inappropriate in some applications. In our model of entry outcomes below, $N_{t}$ is completely determined by factors common knowledge among bidders. HPP point out that in OCS auctions, rivals' joint bidding agreements and participation in follow-up seismic surveys are typically known, and that bidders performing a follow-up survey submit bids on roughly $80 \%$ of the tracts analyzed. Nonetheless, this leaves some room for uncertainty about the level of competition faced. One challenge in relaxing our assumption
} 
In addition, each bidder $i$ knows the signal $S_{i t}$. Let $F_{S V}\left(S_{t}, V_{t} \mid N_{t}, X_{t}, U_{t}\right)$ denote the joint distribution of signals and valuations conditional on $\left(N_{t}, X_{t}, U_{t}\right)$. We make the following standard assumptions on this conditional distribution.

Assumption 2. (i) For all $n \in \operatorname{supp} N_{t} \mid\left(X_{t}, U_{t}\right), F_{S V}\left(S_{t}, V_{t} \mid n, X_{t}, U_{t}\right)$ has a continuously differentiable joint density that is affiliated, exchangeable in the indices $i=1, \ldots, n$, and positive on $(\underline{s}, \bar{s})^{n} \times(\underline{v}, \bar{v})^{n}$; (ii) $E\left[V_{i t} \mid S_{i t}, S_{-i t}, N_{t}, X_{t}, U_{t}\right]$ exists and is strictly increasing in $S_{i t}$.

Because the bidding stage involves a standard affiliated values model, it nests a variety of special cases. With private values, $E\left[V_{i t} \mid S_{i t}, S_{-i t}, N_{t}, X_{t}, U_{t}\right]$ does not depend on $S_{-i t}$. In our setting this is equivalent to bidders' knowing their valuations, i.e., $S_{i t}=V_{i t}=w\left(S_{i t} ; N_{t}, X_{t}, U_{t}\right)$. When $E\left[V_{i t} \mid S_{i t}, S_{-i t}, N_{t}, X_{t}, U_{t}\right]$ depends on $S_{-i t}$, we have common values (or interdependent values). A special case of the common values model is that of pure common values, where $V_{i t}=\bar{V}_{t}$ for all $i$.

A conditional expectation of particular relevance for what follows is

$$
w\left(s_{i t} ; n_{t}, x_{t}, u_{t}\right) \equiv E\left[V_{i t} \mid S_{i t}=\max _{j \neq i} S_{j t}=s_{i t}, N_{t}=n_{t}, X_{t}=x_{t}, U_{t}=u_{t}\right] .
$$

This is a bidder's expected value of winning the auction conditional on all common knowledge information, the observed private signal, and the event (typically counterfactual) that this signal ties for the highest among all bidders at the auction. This expectation plays an important role in the theory because, when equilibrium bidding strategies turn out to be strictly increasing in signals, tying for the highest signal means that even arbitrarily small deviations from one's equilibrium bid will change the identity of the winner. We therefore refer to $w\left(s_{i t} ; n_{t}, x_{t}, u_{t}\right)$ as bidder $i$ 's "pivotal expected value" at auction $t$. Pivotal expected values also play a central role in our

is the need to specify precisely the information available to bidders when forming expectations of the competition. This is an important topic that we leave to future work. 
strategy for discriminating between private values and common values.

We impose the following restriction on how the auction characteristics $\left(X_{t}, U_{t}\right)$ affect bidder valuations.

Assumption 3. (i) $V_{i t}=\Gamma\left(X_{t}, U_{t}\right) V_{i t}^{0}$; (ii) conditional on $N_{t},\left(V_{1 t}^{0}, \ldots, V_{N_{t} t}^{0}, S_{1 t}, \ldots, S_{N_{t} t}\right)$ is independent of $\left(X_{t}, U_{t}\right)$; (iii) $\Gamma$ is bounded and weakly increasing in $U_{t}$.

Assumption 3 is an index restriction requiring multiplicative separability in $\left(X_{t}, U_{t}\right)$ and weak monotonicity in $U_{t} \cdot{ }^{11}$ An assumption of multiplicative (or additive) separability has often been relied upon in the auctions literature, including for identification in other settings with unobserved heterogeneity. Our identification result will rely on this assumption as well ${ }^{12}$ Without further loss, we normalize the scale of $\Gamma$ relative to that of $V_{i t}^{0}$ by taking an arbitrary point $x^{0} \in \mathbb{X}$ and setting

$$
\Gamma\left(x^{0}, 0\right)=1 .
$$

We assume initially that the auction is conducted without a binding reserve price, although below we consider an extension allowing a random reserve price. Under Assumption 2, the auction stage of our model admits a unique Bayesian Nash equilibrium in weakly increasing strategies; these strategies, which we denote by $\beta\left(\cdot ; X_{t}, U_{t}, N_{t}\right):[\underline{s}, \bar{s}] \rightarrow \mathbb{R}$, are symmetric and strictly increasing ${ }^{13}$ Let the random variable $B_{i t}=\beta\left(S_{i t} ; X_{t}, U_{t}, N_{t}\right)$ denote the equilibrium bid of bidder $i$ in auction $t$, with $B_{t}$ denoting $\left(B_{1 t}, \ldots, B_{N_{t} t}\right)$.

\footnotetext{
${ }^{11}$ Without a distributional restriction like that in part (ii) of the assumption, part (i) would have no content. And because more "desirable" realizations of the unobservable state can be labeled with larger values, the monotonicity restriction in (iii) only rules out variation with $X_{t}$ in the partial order on the unobservable implied by desirability (see also Example 1 and Appendix A.

${ }^{12}$ For for what follows it is sufficient that the conditional expectations $E\left[V_{i t} \mid S_{t}, X_{t}, U_{t}, N_{t}\right]$ take the multiplicatively separable form. This weaker condition will be more natural when these ex ante conditional expectations are positive even though $V_{i t}$ may take negative values.

${ }^{13}$ See Theorem 2.1 in Athey and Haile (2007) and the associated references. Milgrom and Weber (1982) characterize the equilibrium strategies.
} 
A useful fact is that the separability required by Assumption 3 is is inherited by the equilibrium bidding strategies 14 Thus, under Assumptions 2 and 3 we may write

$$
\beta\left(S_{i t} ; X_{t}, U_{t}, N_{t}\right)=\Gamma\left(X_{t}, U_{t}\right) \beta^{0}\left(S_{i t} ; N_{t}\right)
$$

where $\beta^{0}$ denotes the symmetric Bayesian Nash equilibrium bidding strategy for a (possibly hypothetical) auction $t$ at which $\Gamma\left(X_{t}, U_{t}\right)=\Gamma\left(x^{0}, 0\right)=1$. Following HHS, we refer to $B_{i t}^{0}=\beta^{0}\left(S_{i t} ; N_{t}\right)$ and $V_{i t}^{0}$ as "homogenized" bids and valuations.

We link the model of a single auction to the observed sample through Assumption 4. Given Assumption 3, this is the standard assumption that auctions are i.i.d. conditional on auction characteristics, $\left(N_{t}, X_{t}, U_{t}\right)$. However, we do not require $U_{t}$ to be independent across auctions.

Assumption 4. $\left(V_{t}^{0}, S_{t}\right) \Perp\left(V_{t^{\prime}}^{0}, S_{t^{\prime}}\right)$ for $t^{\prime} \neq t$.

Finally, we specify the outcome of the entry stage by supposing that the number of bidders at auction $t$ satisfies

$$
N_{t}=\eta\left(X_{t}, Z_{t}, U_{t}\right)
$$

for some function $\eta$ that is weakly increasing in $U_{t}$. Formally, (3) is an assumed reduced form for the entry outcome. The weak monotonicity requirement links the interpretation of the unobservable in the entry and bidding stages: unobservables that make the good for sale more valuable also encourage more entry. The new variable $Z_{t}$ in (3) is an exogenous auction-specific observable that affects bidder entry but is otherwise excludable from the auction model, as formalized in Assumption 5.

Assumption 5. (i) $Z_{t} \Perp U_{t} \mid X_{t}$; (ii) $Z_{t} \Perp\left(S_{t}, V_{t}^{0}\right) \mid N_{t}$.

\footnotetext{
${ }^{14}$ See, e.g., HHS, Athey and Haile (2007), or Krasnokutskaya (2011).
} 
The following example, discussed more fully in Appendix A, describes one fully specified two-stage game leading to the structure assumed above.

Example 1. Consider a model of entry and bidding for an OCS oil and gas lease, where a standard simultaneous move entry stage à la Berry (1992) precedes a competitive bidding stage à la Milgrom and Weber (1982). Players in the game are firms in the industry. The tract offered for lease is associated with observables $X_{t}$, which includes (among other relevant covariates) the number of active leases on neighbor tracts and the sets of bidders for those leases.15 The active neighbor leases are owned by $Z_{t}$ distinct neighbor firms. Tract-level unobservables are denoted by $E_{t}$. $E_{t}$ may have arbitrary dimension, may be correlated with $X_{t}$, and may be spatially correlated. The characteristics $X_{t}$ and $E_{t}$ are assumed to scale valuations (multiplicatively) through a bounded index $\lambda\left(X_{t}, E_{t}\right)$. Firms play a two-stage game. They first choose simultaneously whether to enter, with each entering firm $i$ incurring a signal acquisition cost $c_{i}\left(X_{t}\right)$. Signal acquisition costs are common knowledge and lower for neighbor firms than other (non-neighbor) firms ${ }^{16}$ Entrants learn their private signals and the number of entrants, then participate in a first-price sealed bid auction with symmetric affiliated values. Appendix A shows that all pure strategy perfect Bayesian equilibria (with weakly increasing strategies in the bidding stage) can be represented by the model and assumptions above. This representation is obtained by defining $U_{t}=F_{\lambda}\left(\lambda\left(x_{t}, E_{t}\right) \mid x_{t}\right)$, where $F_{\lambda}(\cdot \mid x)$ is the CDF of the random variable $\lambda\left(x, E_{t}\right)$. Observe that in this case the distribution of $U_{t}$ does not vary with $X_{t}$, although its interpretation does. ${ }^{17}$

\footnotetext{
${ }^{15}$ Tracts $s$ and $t$ are defined to be neighbors if their boundaries coincide at some point.

${ }^{16}$ This structure generalizes that in Hendricks and Porter (1988), where neighbors obtain a private signal for free but non-neighbors face an infinite cost of signal acquisition.

${ }^{17}$ In that case, knowledge of the function $\Gamma$ will not be sufficient to characterize the effects of a ceteris paribus change in $X_{t}$ on bidder valuations. See the additional discussion in Appendix $\mathrm{A}$.
} 


\section{Nonparametric Identification}

In this section we develop sufficient conditions for identification of the entry model, the index function $\Gamma$, and key features of the bidding model. We address each of these in turn. Throughout we assume that the observables include $X_{t}, Z_{t}, N_{t}$ and $B_{t} .^{18}$ Let $\mathbb{Y}$ denote the support of $\left(X_{t}, Z_{t}\right)$, and let $\mathbb{Y}(n)$ denote the support of $\left(X_{t}, Z_{t}\right)$ conditional on $N_{t}=n$. Let $\underline{n} \geq 0$ denote the minimum value in the support of $N_{t}$; let $\bar{n}$ denote the maximum. Recalling (1), for convenience we take $x^{0}$ such that for some $z$ we have $\left(x^{0}, z\right) \in \mathbb{Y}(\underline{n})$.

\subsection{Identification of the Entry Model}

We show identification of the entry model under the following regularity condition on the support of $N_{t} \mid\left(X_{t}, Z_{t}\right)$.

Assumption 6. For all $(x, z) \in \mathbb{Y}$, there exist $\underline{n}(x, z)$ and $\bar{n}(x, z)$ such that $\eta\left(x, z, U_{t}\right)$ has support $(\underline{n}(x, z), \underline{n}(x, z)+1, \ldots, \bar{n}(x, z))$.

Given Assumption 6, for any $(x, z) \in \mathbb{Y}$, the function $\eta(x, z, \cdot)$ is characterized by the thresholds

$$
\tau_{\underline{n}(x, z)-1}(x, z) \leq \tau_{\underline{n}(x, z)}(x, z) \leq \ldots \leq \tau_{\bar{n}(x, z)},
$$

where

$$
\tau_{\underline{\underline{n}}(x, z)-1}(x, z)=0, \quad \tau_{\bar{n}(x, z)}(x, z)=1,
$$

and for $n=\{\underline{n}(x, z), \ldots, \bar{n}(x, z)\}$,

$$
\tau_{n-1}(x, z)=\inf \{u \in[0,1]: \eta(x, z, u) \geq n\}
$$

\footnotetext{
${ }^{18}$ In some applications, data may available only for auctions attracting at least one bidder. In Appendix A we show that, within the fully specified model of Example1, our maintained assumptions and analysis remain valid in the presence of such sample selection. Our examination of the OCS auction data will exploit this result.
} 
With this observation, identification of $\eta$ follows easily.

Theorem 1. Under Assumptions 1, 6, $\eta$ is identified.

Proof. Take arbitrary $(x, z) \in \mathbb{Y}$. For each $n \in\{\underline{n}(x, z), \ldots, \bar{n}(x, z)\}$,

$$
\begin{aligned}
\operatorname{Pr}\left(N_{t}=n \mid X_{t}=x, Z_{t}=z\right) & =\operatorname{Pr}\left(\tau_{n-1}(x, z) \leq U_{t} \leq \tau_{n}(x, z) \mid X_{t}=x, Z_{t}=z\right) \\
& =\tau_{n}(x, z)-\tau_{n-1}(x, z) .
\end{aligned}
$$

The probabilities on the left are observed. Thus, using (4), equation (5) can be solved iteratively for the unknown thresholds $\tau_{n}(x, z)$ starting from $n=\underline{n}(x, z)$.

Identification of $\eta$ determines the effects of $Z_{t}$ on bidder entry and provides bounds $\tau_{n_{t}-1}\left(x_{t}, z_{t}\right)$ and $\tau_{n_{t}}\left(x_{t}, z_{t}\right)$ on the realization of each unobservable $U_{t}$. As shown in the following corollary (proved in Appendix B), it also determines the distribution of $U_{t}$ conditional on $\left(X_{t}, N_{t}\right)$.

Corollary 1. Under Assumptions 1 , 6 , the distribution of $U_{t} \mid\left(X_{t}, N_{t}\right)$ is identified.

\subsection{Identification of the Index Function}

Define

$$
\gamma(x, u)=\ln \Gamma(x, u) .
$$

We first provide conditions sufficient to identify $\gamma(x, u)$ at each $x \in \mathbb{X}$ and $u \in \mathcal{U}_{x}$, where

$$
\mathcal{U}_{x}=\bigcup_{\substack{z:(x, z) \in \mathbb{Y} \\ n \in \operatorname{supp} \eta\left(x, z, U_{t}\right)}}\left\{\tau_{n-1}(x, z), \tau_{n}(x, z)\right\} .
$$

We then give additional conditions guaranteeing that $\mathcal{U}_{x}=[0,1]$ for each $x$. We begin with the following result, whose proof illustrates a key argument. 
Lemma 1. Under Assumptions $1 \sqrt{6}$, for all $n \geq \underline{n}$, all $(x, z) \in \mathbb{Y}(n)$, and all $\left(x^{\prime}, z^{\prime}\right) \in$ $\mathbb{Y}(n)$, the differences $\gamma\left(x^{\prime}, \tau_{n}\left(x^{\prime}, z^{\prime}\right)\right)-\gamma\left(x, \tau_{n}(x, z)\right)$ and $\gamma\left(x^{\prime}, \tau_{n-1}\left(x^{\prime}, z^{\prime}\right)\right)-\gamma\left(x, \tau_{n-1}(x, z)\right)$ are identified.

Proof. By (2) and monotonicity of the equilibrium bid function,

$$
\inf \left\{\ln B_{i t} \mid N_{t}=n, X_{t}=x, Z_{t}=z\right\}=\gamma\left(x, \tau_{n-1}(x, z)\right)+\ln \beta^{0}(\underline{s} ; n) .
$$

So under Assumptions 1 , for any $n$ and all $(x, z)$ and $\left(x^{\prime}, z^{\prime}\right)$ in $\mathbb{Y}(n)$, the differences

$$
\gamma\left(x^{\prime}, \tau_{n-1}\left(x^{\prime}, z^{\prime}\right)\right)-\gamma\left(x, \tau_{n-1}(x, z)\right)
$$

are identified 19 Similarly, since

$$
\sup \left\{\ln B_{i t} \mid N_{t}=n, X_{t}=x, Z_{t}=z\right\}=\gamma\left(x, \tau_{n}(x, z)\right)+\ln \beta^{0}(\bar{s} ; n),
$$

we obtain identification of the differences

$$
\gamma\left(x^{\prime}, \tau_{n}\left(x^{\prime}, z^{\prime}\right)\right)-\gamma\left(x, \tau_{n}(x, z)\right)
$$

for all $n$ and all $(x, z)$ and $\left(x^{\prime}, z^{\prime}\right)$ in $\mathbb{Y}(n)$.

Thus far we have not imposed any requirement on the support of $Z_{t}$ or its effect on entry outcomes. Below we will do so in order to obtain point identification of $\gamma$. However, even in the case that no instrument is available, Theorem 1, Corollary 1, and Lemma 1 still hold. And once $\gamma$ is known, $Z_{t}$ plays no further role in the identification results. Thus, while we rely on an instrument to obtain point identification, this reliance is formally limited to ensuring that we can move from the partial identification

\footnotetext{
${ }^{19}$ Because $\beta^{0}(\underline{s} ; n)=E\left[V_{i t}^{0} \mid S_{j t}=\underline{s}, j=1, \ldots, n\right]$ and $\beta^{0}(s ; n) \leq E\left[V_{i t}^{0} \mid S_{j t}=s, j=1, \ldots, n\right]$ for all $s$ (see, e.g., Milgrom and Weber $(1982))$, both $\beta^{0}(\underline{s} ; n)$ and $\beta^{0}(\bar{s} ; n)$ are finite under Assumption 2 .
} 
of $\gamma$ provided by Lemma 1 to point identification of $\gamma$.

As a step toward point identification, we introduce two additional assumptions. These allow us to show that the first differences obtained above can be differenced again, cancelling common terms, to obtain a set of first differences sufficient to pin down the value of the index $\gamma(x, u)$ at all $x$ and $u \in \mathcal{U}_{x}$.

Assumption 7. For all $n \in\{\underline{n}+1, \underline{n}+2, \ldots, \bar{n}\}, \mathbb{Y}(n-1) \cap \mathbb{Y}(n)$ is nonempty.

Assumption 8. There exists $n^{*}$ such that

(i) $\forall n \in\left\{\underline{n}, \ldots, n^{*}\right\}, \mathbb{Y}$ contains points $(x(n), z(n))$ and $(x(n), \hat{z}(n))$ such that $\underline{n}(x(n), z(n))=n$ and $\underline{n}(x(n), \hat{z}(n))=n+1 ;$ and

(ii) $\forall n \in\left\{n^{*}, \ldots, \bar{n}\right\}, \mathbb{Y}$ contains points $\left(x^{\prime}(n), z^{\prime}(n)\right)$ and $\left(x^{\prime}(n), \hat{z}^{\prime}(n)\right)$ such that $\bar{n}\left(x^{\prime}(n), z^{\prime}(n)\right)=n$ and $\bar{n}\left(x^{\prime}(n), \hat{z}^{\prime}(n)\right)=n-1$.

Assumption 7 requires variation in $U_{t}$ that produces local variation in entry. For example, this rules out trivial cases in which $U_{t}$ has no effect on $N_{t}$. Assumption 8 requires variation in the instrument $Z_{t}$ that can induce local variation in the support of the entry outcomes, at least at some values of $X_{t}$.

We prove the following results in Appendix $B$.

Lemma 2. Under Assumptions 1 8, for all $n \geq \underline{n}$, all $(x, z) \in \mathbb{Y}(n)$, and all $\left(x^{\prime}, z^{\prime}\right) \in$ $\mathbb{Y}(n), \gamma\left(x, \tau_{n}(x, z)\right)-\gamma\left(x^{\prime}, \tau_{n-1}\left(x^{\prime}, z^{\prime}\right)\right)$ is identified.

Lemma 3. Under Assumptions 1 8, for all $n \geq \underline{n}$ and all $(x, z) \in \mathbb{Y}(n)$, the values of $\gamma\left(x, \tau_{n-1}(x, z)\right)$ and $\gamma\left(x, \tau_{n}(x, z)\right)$ are identified.

By Theorem 1, the values of $\tau_{n-1}(x, z)$ and $\tau_{n}(x, z)$ are known for all $n$ and $(x, z) \in \mathbb{Y}(n)$. Thus, Lemma 3 demonstrates identification of $\gamma(x, u)$ at each $x \in \mathbb{X}$ and $u \in \mathcal{U}_{x}$. In general, this may still deliver only partial identification of the index function $\gamma$, so that in practice one may rely on parametric structure to interpolate between the points $\left\{x \in \mathbb{X}, u \in \mathcal{U}_{x}\right\}$ at which $\gamma(x, u)$ is nonparametrically point 
identified. However, the following conditions are sufficient to ensure that no such interpolation is necessary.

Assumption 9. (a) For all $x \in \mathbb{X}, \operatorname{supp} Z_{t} \mid X_{t}=x$ is connected.

(b) For all $(x, z, u) \in \mathbb{Y} \times(0,1)$ and all $\delta>0$ such that $(u-\delta, u+\delta) \subset(0,1)$, there exists $\epsilon>0$ such that if $\left\|z^{\prime}-z\right\|<\epsilon$ then $\eta\left(x, z^{\prime}, u^{\prime}\right)=\eta(x, z, u)$ for some $u^{\prime} \in(u-\delta, u+\delta)$.

Assumption 10. For every $x \in \mathbb{X}$ there exists a finite partition $0=\tau^{0}(x)<\tau^{1}(x)<$ $\cdots<\tau^{K(x)}(x)=1$ of the unit interval such that for each $k=1, \ldots, K(x)$ and some $z(k), z^{\prime}(k) \in \operatorname{supp} Z_{t} \mid X_{t}=x, \eta\left(x, z(k), \tau^{k-1}(x)\right)>\eta\left(x, z^{\prime}(k), \tau^{k}(x)\right)$.

Assumption 9 rules out discrete instruments and requires a type of continuous substitution between $Z_{t}$ and $U_{t}$ in the "production" of bidder entry: it must be possible to offset the effect (on entry) of a small change in $Z_{t}$ with a small change in $U_{t}$. Assumption 10 requires that variation in $Z_{t}$ have sufficient effect on participation to offset some discrete variation in the unobservable $U_{t}$. A sufficient condition is that for each $x$ there exist $z$ and $z^{\prime}$ such that $\eta\left(x, z, \tau_{n-1}(x, z)\right)>\eta\left(x, z^{\prime}, \tau_{n}(x, z)\right)$ for all $n \in\{\underline{n}(x, z), \ldots, \bar{n}(x, z)\}$; in this case, the set $\left\{\tau_{\underline{n}(x, z)-1}, \ldots, \tau_{\bar{n}(x, z)}\right\}$ could define the partition $\tau^{0}(x)<\tau^{1}(x)<\cdots<\tau^{K}(x)$.

The following lemma, whose proof is provided in Appendix B, leads us to the point identification of $\gamma$ (and therefore $\Gamma$ ) demonstrated in Theorem 2.

Lemma 4. Under Assumptions 1 9, $\tau_{n-1}\left(X_{t}, Z_{t}\right)$ is continuous in $Z_{t}$ on the preimage of $(0,1)$.

Theorem 2. Under Assumptions 1 10, $\Gamma$ is identified on $\mathbb{X} \times[0,1]$.

Proof. We need only show that $\mathcal{U}_{x}=[0,1]$ for each $x \in \mathbb{X}$. For arbitrary $x \in$ $\mathbb{X}$, let $0=\tau^{0}(x)<\tau^{1}(x)<\cdots<\tau^{K(x)}(x)=1$ be as in Assumption 10 . Take any $k \in\{1,2, \ldots, K(x)\}$ and let $z=z(k)$ and $z^{\prime}=z^{\prime}(k)$ be as in Assumption 
10. Let $n=\eta\left(x, z, \tau^{k-1}(x)\right)$. Because $\eta\left(x, z, \tau^{k-1}(x)\right)>\eta\left(x, z^{\prime}, \tau^{k}(x)\right)$, we have $\eta\left(x, z^{\prime}, \tau^{k}(x)\right)<n$ and, therefore,

$$
\tau_{n-1}(x, z) \leq \tau^{k-1}(x)<\tau^{k}(x) \leq \tau_{n-1}\left(x, z^{\prime}\right)
$$

Because the continuous image of a connected set is connected, Lemma 4 and Assumption 9 (part (a)) imply that for every $\tilde{\tau} \in\left[\tau^{k-1}(x), \tau^{k}(x)\right]$ there exists $z^{\tilde{\tau}}$ such that $\tau_{n-1}\left(x, z^{\tilde{\tau}}\right)=\tilde{\tau}$.

\subsection{Identification of the Bidding Model}

We now demonstrate identification of the joint distribution of the pivotal expected values $\left(w\left(S_{1 t} ; n, x, u\right), \ldots, w\left(S_{n t} ; n, x, u\right)\right)$ for all $x \in \mathbb{X}, u \in[0,1]$, and $n$ in the support of $N_{t} \mid\left\{X_{t}=x, U_{t}=u\right\}$. For a private values model, where $V_{i t}=S_{i t}$, this is equivalent to identification of the joint distribution of bidder valuations conditional on $\left(X_{t}, N_{t}, U_{t}\right)$. Thus, Theorem 3 below demonstrates identification of the affiliated private values model. Without the restriction to private values, our result here provides an important form of partial identification 20 For our empirical application, for example, this is sufficient to allow us to test the hypotheses of equilibrium bidding in the affiliated values model and to test the hypothesis of private values against the alternative of common values.

Let

$$
G_{M \mid B}(m \mid b, x, u, n)=\operatorname{Pr}\left(\max _{j \neq i} B_{j t} \leq m \mid B_{i t}=b, X_{t}=x, U_{t}=u, N_{t}=n\right)
$$

and let $g_{M \mid B}(m \mid b, x, u, n)$ denote the associated conditional density (guaranteed to

\footnotetext{
${ }^{20}$ Without additional information or structure, common values models are not identified from bidding data. See, e.g., Laffont and Vuong (1993) and Athey and Haile (2002).
} 
exist by Assumption 2 and strict monotonicity of the equilibrium bid function). Following Laffont and Vuong (1993), Guerre, Perrigne, and Vuong (2000), and Li, Perrigne, and Vuong (2000, 2002), one can characterize the relationship between each realized $w\left(s_{i t} ; n_{t}, x_{t}, u_{t}\right)$ and the associated equilibrium bid $b_{i t}=\beta\left(s_{i t} ; x_{t}, u_{t}, n_{t}\right)$ in terms of the joint distribution of equilibrium bids. In particular, each $b_{i t}$ must satisfy the first-order condition 21

$$
w\left(s_{i t} ; n_{t}, x_{t}, u_{t}\right)=b_{i t}+\frac{G_{M \mid B}\left(b_{i t} \mid b_{i t}, x_{t}, u_{t}, n_{t}\right)}{g_{M \mid B}\left(b_{i t} \mid b_{i t}, x_{t}, u_{t}, n_{t}\right)} .
$$

Although this equation expresses the pivotal expected value $w\left(s_{i t} ; n_{t}, x_{t}, u_{t}\right)$ as a functional of a conditional distribution of bids, the presence of $u_{t}$ on the right-hand side creates challenges. Because realizations of $U_{t}$ are not observable or identified, one cannot directly condition on them to identify the functions $G_{M \mid B}$ and $g_{M \mid B}$. This precludes obtaining identification directly from (8). With the preceding results, however, we can overcome this problem.

Observe that, like valuations and bids, the pivotal expected values $w\left(s_{i t} ; n_{t}, x_{t}, u_{t}\right)$ will have the separable structure

$$
w\left(s_{i t} ; n_{t}, x_{t}, u_{t}\right)=w^{0}\left(s_{i t} ; n_{t}\right) \Gamma\left(x_{t}, u_{t}\right),
$$

where

$$
w^{0}\left(s_{i t} ; n_{t}\right) \equiv E\left[V_{i t}^{0} \mid S_{i t}=\max _{j \neq i} S_{j t}=s_{i t}, N_{t}=n_{t}\right] .
$$

We will refer to $w^{0}\left(s_{i t} ; n_{t}\right)$ as bidder $i$ 's "homogenized pivotal expected value" at auction $t$. The first-order condition (8) can then be written as

$$
w^{0}\left(s_{i t} ; n_{t}\right)=b_{i t}^{0}+\frac{G_{M^{0} \mid B^{0}}\left(b_{i t}^{0} \mid b_{i t}^{0}, n_{t}\right)}{g_{M^{0} \mid B^{0}}\left(b_{i t}^{0} \mid b_{i t}^{0}, n_{t}\right)}
$$

\footnotetext{
${ }^{21}$ See, e.g., Athey and Haile $\sqrt{2007)}$ for a derivation in the affiliated values model.
} 
where

$$
G_{M^{0} \mid B^{0}}\left(m \mid b, n_{t}\right)=\operatorname{Pr}\left(\max _{j \neq i} B_{j t}^{0} \leq m \mid B_{i t}^{0}=b, N_{t}=n\right)
$$

and $g_{M^{0} \mid B^{0}}\left(m \mid b, n_{t}\right)$ is the associated conditional density.

With the following regularity condition, Theorem 2 and a standard deconvolution argument will yield identification of the joint distribution of $\left(B_{1 t}^{0}, \ldots, B_{n t}^{0}\right)$ for all $n$.

Assumption 11. For some $x \in \mathbb{X}$ the random variable $\gamma\left(x, U_{t}\right)$ has nonzero characteristic function almost everywhere.

Lemma 5. Under Assumptions 111 , conditional on any $N_{t}=n$, the joint density of $\left(B_{1 t}^{0}, \ldots, B_{n t}^{0}\right)$ is identified.

Proof. Fix $N_{t}=n$ and $X_{t}=x$, where $x$ is as in Assumption 11 . Let $\tilde{B}_{i t}=\ln \left(B_{i t}\right)$ and $\tilde{B}_{i t}^{0}=\ln \left(B_{i t}^{0}\right)$. By Assumption 3 .

$$
\tilde{B}_{i t}=\tilde{B}_{i t}^{0}+\gamma\left(x, U_{t}\right)
$$

and, furthermore, $\tilde{B}_{i t}^{0}$ and $\gamma\left(x, U_{t}\right)$ are independent conditional on $N_{t}$. Let $\psi_{\tilde{B}}$ denote the characteristic function of the $\log$ bids $\left(\tilde{B}_{1 t}, \ldots, \tilde{B}_{n t}\right)$ conditional on $X_{t}=x$ and $N_{t}=n$. Let $\psi_{\gamma}$ denote the characteristic function of $\gamma\left(x, U_{t}\right)$ conditional on $N_{t}=n$. For $\left(r_{1}, \ldots, r_{n}\right) \in \mathbb{R}^{n}$ we then have

$$
\psi_{\tilde{B}}\left(r_{1}, \ldots, r_{n}\right)=\psi_{\tilde{B}^{0}}\left(r_{1}, \ldots, r_{n}\right) \psi_{\gamma}\left(r_{1}+\cdots+r_{n}\right)
$$

where $\psi_{\tilde{B}^{0}}$ is the characteristic function of the log homogenized bids $\left(\tilde{B}_{1 t}^{0}, \ldots, \tilde{B}_{n t}^{0}\right)$ conditional on $N_{t}=n$. Since the distribution of $U_{t} \mid\left(X_{t}, N_{t}\right)$ is known (Corollary 1 ) and $\gamma$ is a known function (Theorem 2), $\psi_{\gamma}$ is known. So under Assumption 11 the equation

$$
\psi_{\tilde{B}^{0}}\left(r_{1}, \ldots, r_{n}\right)=\frac{\psi_{\tilde{B}}\left(r_{1}, \ldots, r_{n}\right)}{\psi_{\gamma}\left(r_{1}+\cdots+r_{n}\right)}
$$


uniquely determines $\psi_{\tilde{B}^{0}}\left(r_{1}, \ldots, r_{n}\right)$ for almost all $\left(r_{1}, \ldots, r_{n}\right)$. By continuity of characteristic functions this yields identification of $\psi_{\tilde{B}^{0}}$, implying identification of the joint density of $\left(\tilde{B}_{1 t}^{0}, \ldots, \tilde{B}_{n t}^{0}\right){ }^{22}$ The result then follows.

This leads directly to our main identification result.

Theorem 3. Let Assumptions 111 hold. Then for all $x \in \mathbb{X}, u \in[0,1]$, and $n \geq 2$ in the support of $N_{t} \mid\left\{X_{t}=x\right\}$, the joint distribution of $\left(w\left(S_{1 t} ; n, x, u\right), \ldots, w\left(S_{n t} ; n, x, u\right)\right)$ is identified.

Proof. Fix $n$. From (10), we have

$$
w^{0}\left(S_{i t} ; n\right)=\xi\left(B_{i t}^{0} ; n\right) \equiv B_{i t}^{0}+\frac{G_{M^{0} \mid B^{0}}\left(B_{i t}^{0} \mid B_{i t}^{0}, n\right)}{g_{M^{0} \mid B^{0}}\left(B_{i t}^{0} \mid B_{i t}^{0}, n\right)} .
$$

By Lemma 5, the joint distribution $\left(\xi\left(B_{1 t}^{0} ; n\right), \ldots, \xi\left(B_{n t}^{0} ; n\right)\right)$ is known. This implies identification of the joint distribution of $\left(w^{0}\left(S_{1 t} ; n\right), \ldots, w^{0}\left(S_{n t} ; n\right)\right)$. The result then follows immediately from $(9)$ and Theorem 2.

\section{Estimation}

We propose a two-stage semiparametric estimation strategy. The first stage involves semiparametric sieve quasi-maximum likelihood estimation (QMLE) of the entry thresholds $\tau_{\ell}(x, z)$, the index function $\gamma$, and the joint distributions of homogenized equilibrium bids. In the second stage, for each level of bidder entry, we estimate the joint distribution of homogenized pivotal expected values by plugging draws from the estimated distribution of homogenized bids into the auction first-order condition and constructing the empirical distribution of the resulting pseudo-sample.

\footnotetext{
${ }^{22}$ Because the same argument holds at all $x \in \mathbb{X}$ such that the characteristic function of $\gamma\left(x, U_{t}\right)$ is nonvanishing a.s., the argument demonstrating Lemma 5 may often yield overidentification.
} 


\subsection{Stage 1: Sieve-QMLE}

Let $\theta_{\tau}$ denote the parameters of the entry model, $\theta_{\gamma}$ the parameters of the index function $\gamma$, and $\theta_{B}$ the parameters of the joint distributions of log homogenized bids. Let

$$
L_{1 t}\left(n_{t} ; \theta_{\tau}\right)=\operatorname{Pr}\left(N_{t}=n_{t} \mid X_{t}=x_{t}, Z_{t}=z_{t} ; \theta_{\tau}\right)
$$

denote the (conditional on $\left.\left(x_{t}, z_{t}\right)\right)$ likelihood for the entry outcome in auction $t$. Let

$$
L_{2 t}\left(b_{t} \mid n_{t} ; \theta_{\gamma}, \theta_{B}, \theta_{\tau}\right)
$$

denote the likelihood of the observed bids at auction $t$, conditional on the entry outcome $n_{t}$ (and on $\left.\left(x_{t}, z_{t}\right)\right)$. Defining $\theta=\left(\theta_{\tau}, \theta_{\gamma}, \theta_{B}\right)$, the conditional quasi-likelihood function for the observed outcomes $\left\{\left(n_{t}, b_{t}\right)\right\}_{t=1}^{T}$ can be written

$$
\mathcal{L}(\theta)=\prod_{t} L_{1 t}\left(n_{t} ; \theta_{\tau}\right) L_{2 t}\left(b_{t} \mid n_{t} ; \theta_{\gamma}, \theta_{B}, \theta_{\tau}\right)
$$

We give details of our empirical specification and the two components of the quasilikelihood in sections 4.1.1 and 4.1.2 below. Estimates of the parameter vector $\theta$ can be obtained by maximizing $\mathcal{L}(\theta)$. Because $\theta_{\tau}$ is identified from the entry outcomes alone, it is also possible to split the QMLE stage, first maximizing $\prod_{t} \mathcal{L}_{1 t}\left(\theta_{\tau}\right)$ to estimate $\theta_{\tau}$, then maximizing $\prod_{t} L_{2 t}\left(\theta_{\gamma}, \theta_{B}, \hat{\theta}_{\tau}\right)$ conditional on $\hat{\theta}_{\tau}$. In our data, the two approaches yield very similar estimates. However, because we found the two-step QMLE procedure to be more stable numerically, below we will report results using the two-step version of the QMLE ${ }^{24}$

Consistency can be confirmed by adapting the results of White and Wooldridge

\footnotetext{
${ }^{23}$ Recall that we permit spatial dependence.

${ }^{24}$ With one-step QMLE, standard optimizers failed to find a maximum in many of the bootstrap replications. Monte Carlo simulations suggest that the two-step procedure performs well at moderate sample sizes.
} 
(1991) for sieve-extremum estimators with weakly dependent time series data to the case of weak spatial dependence. 25 To conduct inference, we use a nonparametric block bootstrap procedure that captures both dependence among bids within an auction and spatial dependence between the unobservables $U_{t}$ across auctions. Specifically, we resample auctions with replacement, taking all bids from the selected auction, and including in the bootstrap sample all auctions on neighbor tracts as well. Following HPP, we resample weighting auctions by factors inversely proportional to the number of auctions in the neighborhood of the tract.26

\subsubsection{Entry Thresholds}

Our entry model above reduces to an ordered response model where, given $X_{t}=x$ and $Z_{t}=z$, we have $N_{t}=n$ if and only if $U_{t} \in\left(\tau_{n-1}(x, z), \tau_{n}(x, z)\right)$. Given any strictly increasing univariate $\mathrm{CDF} H$ we can rewrite this as

$$
\left\{N_{t}=n \mid X_{t}=x, Z_{t}=z\right\} \Longleftrightarrow\left\{A_{t} \in\left(\alpha_{n-1}(x, z), \alpha_{n}(x, z)\right)\right\}
$$

where $A_{t} \sim H$ and $\alpha_{n}(x, z)=H^{-1}\left(\tau_{n}(x, z)\right)$.

We specify a linear threshold function

$$
\alpha_{n}(x, z)=\alpha_{n}-x \alpha_{x}-z \alpha_{z}
$$

\footnotetext{
${ }^{25}$ In particular, we represent tract locations by points in $\mathbb{Z}^{2}$. Then, under a standard "expanding domain" asymptotics, White and Wooldridge's uniform consistency result for stationary $\alpha$-mixing time series data (Corollary 2.6) can be extended using a Bernstein-type inequality for $\alpha$-mixing random fields on $\mathbb{Z}^{2}$ (e.g., Yao (2003)).

${ }^{26}$ Similar results are obtained without weighting. Applying the results of van der Vaart and Wellner (1996) and Lahiri (2003), one can verify validity of this procedure when we interpret our finite-sample estimator as that for a parametric model. General conditions for consistency of bootstrap inference procedures for sieve M-estimators in the i.i.d. setting can be found, e.g., in Ma and Kosorok (2005) and Chen and Pouzo (2009). See also Chen and Liao (2014), and Chen, Liao, and Sun (2014) in the case of time series data.
} 
and specify $H$ as the standard normal CDF, yielding an ordered probit model ${ }^{27}$ In section 7 we consider a variation in which $H$ is specified using a Hermite polynomial series approximation, following Gallant and Nychka (1987). Letting $\theta_{\tau}=$ $\left(\left\{\alpha_{n}\right\}_{n=\underline{n}}^{\bar{n}-1}, \alpha_{x}, \alpha_{z}\right)$, we then have

$$
L_{1 t}\left(n ; \theta_{\tau}\right)=H\left(\alpha_{n}\left(x_{t}, z_{t} ; \theta_{\tau}\right) ; \theta_{\tau}\right)-H\left(\alpha_{n-1}\left(x_{t}, z_{t} ; \theta_{\tau}\right) ; \theta_{\tau}\right)
$$

\subsubsection{Index Function and Homogenized Bid Distribution}

Given our focus on testing for common values, we prioritize flexibility in how the joint distribution and density of bids can vary with $n$ when specifying the second part of the quasi-likelihood. We specify the index function $\gamma$ parametrically as $\gamma\left(\cdot, \cdot ; \theta_{\gamma}\right)$; we will use a linear specification below. The joint density of log homogenized bids is specified semiparametrically, using a parametric copula and a nonparametric (Bernstein polynomial sieve) specification of the common marginal distribution. ${ }^{28}$ Below we specify a Gaussian copula, with separate covariance parameter $\rho_{n}$ for each $n{ }^{29}$

We specify the marginal density of a generic bidder's log homogenized bid in an $n$-bidder auction as

$$
\tilde{g}_{B_{i}^{0}}\left(\tilde{b}^{0} ; \theta_{b}, n\right)=\sum_{j=0}^{m} \theta_{b, n}^{(j)} q_{j, m}\left(\Phi\left(\tilde{b}^{0}\right)\right) \phi\left(\tilde{b}^{0}\right)
$$

\footnotetext{
${ }^{27}$ Thus, although the theoretical model of entry in Example 1 follows Berry $(1992)$, the induced empirical model of the entry stage is similar to that of Bresnahan and Reiss (1991).

${ }^{28}$ See, e.g., Chen, Fan, and Tsyrennikov (2006). Prior work using Bernstein polynomials in estimation of auction models includes Komarova (2017) and Kong (2017a b). Hubbard, Paarsch, and Li (2012) have previously combined a parametric copula and nonparametric (kernel) specification of marginal densities to estimate auction models.

${ }^{29}$ Our use of a parametric copula reflects in part our choice to let the distribution of bids be fully flexible (i.e., even in finite sample) with respect to the number of bidders $n_{t}$. In other applications one might specify a Bernstein copula and account for the effects of $n_{t}$ within the sieve approximation. As we discuss in Appendix E, the Gaussian copula provides a substantial computational advantage when we transform our estimated joint distributions and densities to the conditional distributions and densities that are plugged into bidders' first-order conditions.
} 
where $q_{j, m}(\nu)=\left(\begin{array}{c}m \\ j\end{array}\right) \nu^{j}(1-\nu)^{m-j}$ and $\Phi(\cdot)$ and $\phi(\cdot)$ denote the standard normal distribution and density functions, respectively. Here $m$ is a parameter, growing with the sample size, that determines the order of the Bernstein polynomial approximation. Let $\theta_{b, n}=\left\{\theta_{b, n}^{(j)}\right\}_{j=0}^{m}$. Thus, the parameter vector $\theta_{b}$ in 12 represents $\left\{\theta_{b, n}\right\}_{n=\underline{n}}^{\bar{n}}$.

Because Bernstein polynomials approximate functions with domain $[0,1]$, in 12 . we use Bernstein polynomials to approximate the marginal density of the transformed variable $\Phi\left(\tilde{b}^{0}\right) \cdot 30$ This transformation is useful not only for standardizing the domain but also for ensuring that the nonparametric estimator will offer sensible approximations even in modest sample sizes. When $m=0$, for example, the distribution of log-bids will be normal. Thus, the nonparametric component of our specification is based on a sequence of approximating models that starts with a natural (lognormal) parametric specification and adds flexibility as permitted by the sample size.

Let $\tilde{G}_{B_{i}^{0}}\left(\tilde{b}^{0} ; \theta_{b}, n\right)$ denote the CDF associated with $\tilde{g}_{B_{i}^{0}}\left(\tilde{b}^{0} ; \theta_{b}, n\right)$. Let $\chi\left(\cdot ; \rho_{n}\right)$ denote the symmetric Gaussian copula density with covariance parameter $\rho_{n}{ }^{31}$ We specify the joint density of the log homogenized bids in $n$-bidder auctions as

$$
\begin{aligned}
& \tilde{g}_{B^{0}}\left(\tilde{b}_{1}^{0}, \ldots, \tilde{b}_{n}^{0} ; \theta_{b, n}, \rho_{n}, n\right)= \\
& \quad \chi\left(\tilde{G}_{B_{i}^{0}}\left(\tilde{b}_{1}^{0} ; \theta_{b, n}, n\right), \ldots, \tilde{G}_{B_{i}^{0}}\left(\tilde{b}_{n}^{0} ; \theta_{b, n}, n\right) ; \rho_{n}\right) \tilde{g}_{B_{i}^{0}}\left(\tilde{b}_{1}^{0} ; \theta_{b, n}, n\right) \ldots \tilde{g}_{B_{i}^{0}}\left(\tilde{b}_{n}^{0} ; \theta_{b, n}, n\right) .
\end{aligned}
$$

\footnotetext{
${ }^{30}$ For estimation purposes, we add intercepts $\gamma_{n_{t}}^{0}$ for each value of $n_{t}$ in the index function $\gamma\left(x_{t}, u_{t} ; \theta_{\gamma}\right)$, implying that in this step we actually estimate joint densities of centered log homogenized bids $\tilde{b}_{t}^{0}-\gamma_{n_{t}}^{0}$. We then adjust each estimated density with the appropriate intercept estimate to obtain the density of (uncentered) log homogenized bids that is relevant to bidders' first-order conditions. When homogenization is performed via OLS, this type of centering procedure is required for consistency (see HHS and Athey and Haile (2007)). In our case this is not essential but offers several practical advantages by ensuring that the log homogenized bids are centered at zero prior to transformation by the normal $\mathrm{CDF}$, ensuring that the location of the estimated bid distribution can move freely with $n_{t}$ (again, prioritizing flexibility with respect to $n_{t}$ ), and freeing the Bernstein coefficients to capture features of the marginal density other than its location.

${ }^{31}$ The symmetric Gaussian copula density $\chi\left(h_{1}, \ldots, h_{n} ; \rho_{n}\right)$ is given by $\frac{1}{|\Upsilon|^{1 / 2}} \exp \left(-\frac{1}{2} \varphi(h)^{\prime}\left(\Upsilon^{-1}-I\right) \varphi(h)\right)$, where $\varphi(h)=\left\{\Phi^{-1}\left(h_{1}\right), \ldots, \Phi^{-1}\left(h_{n}\right)\right\}$ and the matrix $\Upsilon$ has ones on the diagonal and covariance $\rho_{n}$ in all off-diagonal entries.
} 
We discuss computational details in Appendix E.

Letting $\rho=\left\{\rho_{n}\right\}_{n=\underline{n}}^{\bar{n}}$ and $\theta_{B}=\left(\theta_{b}, \rho\right)$, we have

$L_{2 t}\left(b_{t} \mid n_{t} ; \theta\right)=\int_{\tau_{n_{t}-1}\left(x_{t}, z_{t} ; \theta_{\tau}\right)}^{\tau_{n_{t}}\left(x_{t}, z_{t} ; \theta_{\tau}\right)} \frac{\tilde{g}_{b^{0}}\left(\tilde{b}_{1 t}-\gamma\left(x_{t}, u ; \theta_{\gamma}\right), \ldots, \tilde{b}_{n_{t} t}-\gamma\left(x_{t}, u ; \theta_{\gamma}\right) ; \theta_{b, n_{t}}, \rho_{n_{t}}, n_{t}\right)}{\tau_{n_{t}}\left(x_{t}, z_{t} ; \theta_{\tau}\right)-\tau_{n_{t}-1}\left(x_{t}, z_{t} ; \theta_{\tau}\right)} d u$

where $\tau_{n_{t}-1}\left(x_{t}, z_{t} ; \theta_{\tau}\right)$ and $\tau_{n_{t}}\left(x_{t}, z_{t} ; \theta_{\tau}\right)$ denote the bounds on $u_{t}$ implied by the entry model and its parameters $\theta_{\tau}{ }^{32}$ We approximate the integral above by Monte Carlo simulation.

\subsection{Stage 2: Invert Equilibrium First-Order Conditions}

Given the first-stage estimates of the index function $\gamma$ and joint distribution of homogenized bids, estimation of the relevant auction primitives is straightforward and does not involve further use of the data. Here we use the equilibrium first-order condition 10 , which can be written in terms of the distribution of log homogenized bids as

$$
w^{0}\left(s_{i t} ; n_{t}\right)=\exp \left(\tilde{b}_{i t}^{0}\right)\left(1+\frac{\tilde{G}_{M \mid B}\left(\tilde{b}_{i t}^{0} \mid \tilde{b}_{i t}^{0}, n_{t}\right)}{\tilde{g}_{M \mid B}\left(\tilde{b}_{i t}^{0} \mid \tilde{b}_{i t}^{0}, n_{t}\right)}\right),
$$

where $\tilde{G}_{M \mid B}\left(\tilde{b}_{i t}^{0} \mid \tilde{b}_{i t}^{0}, n_{t}\right)$ and $\tilde{g}_{M \mid B}\left(\tilde{b}_{i t}^{0} \mid \tilde{b}_{i t}^{0}, n_{t}\right)$ are, respectively, the CDF and pdf of $\max _{j \neq i} \tilde{B}_{j t}^{0}$ conditional on $\tilde{B}_{i t}^{0}=\tilde{b}_{i t}^{0}$.

For each value of $N_{t}=n$, we transform the estimated joint distributions and densities obtained from stage 1 to construct the conditional distributions and densities appearing (15) (see Appendix E). Then we draw log homogenized bids from their estimated marginal distributions and plug these into (15), yielding pseudo-samples

\footnotetext{
${ }^{32}$ When $n_{t}=1$ one may set $L_{2 t}\left(b_{t} \mid n_{t} ; \theta\right)=1$ by convention, since our baseline model of competitive bidding does not allow us to interpret the quantity $\tilde{b}_{i t}-\gamma\left(x_{t}, u_{t}\right)$ in that case. Alternatively, because we specify different parameters $\theta_{b, n}$ for each value of $n, 14$ gives a correct expression for $L_{2 t}\left(b_{t} \mid n_{t} ; \theta\right)$ in a 1-bidder auction whenever bids in 1-bidder auctions are assumed to inherit the separable structure required of valuations. Below we propose an extension incorporating a random reserve price, where this separability is an implication of equilibrium behavior.
} 
of the vectors $\left(w^{0}\left(s_{1 t} ; n\right), \ldots, w^{0}\left(s_{n t} ; n\right)\right)$ for many simulated auctions $t$. The empirical distribution of these pseudo-draws provides a consistent estimate of the joint distribution of homogenized pivotal expected values for $n$-bidder auction. Although these joint distributions will suffice for our application, the pseudo-draws can also be scaled by the estimated value of the index $\Gamma(x, u)$ in order to estimated the joint distributions of (non-homogenized) pivotal expected values given $X_{t}=x$ and $U_{t}=u$. In a private values setting, for example, this would yield an estimate of the joint distribution of bidder valuations.

\section{OCS Auctions}

We apply our method to study auctions of oil and gas leases in the U.S. Outer Continental Shelf (OCS). Our data cover the period 1954 to 1983. The seller in these auctions was the Mineral Management Service ("MMS"), at that time an agency of the U.S. Department of the Interior. Our goal is to examine these auctions through the lens of our model to assess several fundamental features of the market such as the market power of bidders, whether the data support the hypothesis of equilibrium bidding, the effects of competition on bids and revenues, the significance of unobserved heterogeneity and of correlation among bidders' private information, and whether there is empirical support for the presence of common values.

Features of the OCS auction setting arguably suggest common values (perhaps even pure common values), and these auctions are often cited as examples of a common values setting. While prior empirical work on these auctions has provided suggestive evidence in support of a common values model, Li, Perrigne, and Vuong (2000) have argued for formal testing, while HPP have pointed out that such testing has been precluded by the likely presence of unobserved heterogeneity that affects both expected lease value and the level of bidder participation. 


\section{$5.1 \quad$ Background and Data}

Extensive discussion of the OCS auctions can be found in, e.g., Gilley and Karels (1981), Hendricks and Porter (1988), Hendricks, Porter, and Spady (1989), and HPP.

For a more complete institutional background, we refer readers to that prior work, upon which we rely heavily ourselves. Briefly, however, auctions were held for the right to lease a specified tract for exploration and production of oil, gas, and other minerals. Tracts in the sample typically comprise a rectangular area (a "block") covering 5,000-5,760 acres in the Gulf of Mexico 33 Production on a tract was subject to royalty payments from the leaseholder at a pre-specified rate, usually $1 / 6$. Bids at an auction were offers of an additional up-front "bonus" payment for the right to become the leaseholder.

At a given "sale," many tracts were offered for lease simultaneously through separate first-price sealed bid auctions. No exploratory drilling was permitted prior to the auction, although in some cases exploration and production would already have occurred on adjacent ("neighbor") tracts and would be publicly observable. Bids would also reflect information obtained through evaluation of data from magnetic, gravity, and seismic surveys ${ }^{34}$ Although initial collection of survey data was often funded jointly, firms relied on their own experts for modeling and analysis of the data, and firms often performed follow-up surveys of the tracts on which they intended to bid. Differences in expert assessments of the survey data are likely an important source of heterogeneity in bidder beliefs about the value of a given tract (HPP). These features lead us to treat bidder entry as a decision to acquire a costly signal about the value

\footnotetext{
${ }^{33}$ We limit attention to tracts of at least 4,000 acres. Smaller tracts are typically half-blocks or quarter blocks, where several of our measures of neighborhood characteristics would have interpretations different from those on standard tracts. We also drop auctions with missing values for our covariates.

${ }^{34}$ See, e.g., "G\&G Data Acquisition and Analysis" at https://www.boem.gov/Oil-and-GasEnergy-Program/Resource-Evaluation/Geological-and-Geophysical-Data-Acquisition/GGDataGeophysical-Surveys.aspx, March 24, 2018.
} 
of the tract (recall Example 1).

We have data on all auctions attracting at least one bidder ${ }^{35}$ Table 1 shows the number of auctions in our sample by number of bidders. We do not separate wildcat, development, and drainage tracts; instead, we account directly for the presence of active neighbor leases and neighbor production, and allow for asymmetry between neighbor vs. non-neighbor costs of signal acquisition in a way that generalizes the structure considered in Hendricks and Porter (1988) (see Example 1) [4] Like Aradillas-Lopez, Haile, Hendricks, and Porter (2017), we model bidders as symmetric conditional on acquisition of a signal. Thus, while bidders may decide not to acquire a signal through analysis of the seismic data and may reach different conclusions from such analysis, the technology producing signals is modeled as symmetric across firms.

Table 1: Sample Sizes

\begin{tabular}{c|ccccccccccc}
\hline \hline$n$ & 1 & 2 & 3 & 4 & 5 & 6 & 7 & 8 & 9 & 10 & $11-17$ \\
$T_{n}$ & 814 & 498 & 293 & 229 & 172 & 127 & 100 & 73 & 56 & 56 & 128 \\
\hline
\end{tabular}

$T_{n}$ denotes the number of auctions in our sample with $n$ bidders.

The MMS sometimes announced a small minimum acceptable bid of $\$ 10-\$ 25$ per acre; the MMS also retained the option to reject all bids when it deemed the auction to be noncompetitive. Such rejections were rare in our sample except at auctions attracting only one bid (see section 7.1). We initially treat both the announced minimum bid and the MMS bid rejection policy as nonbinding, as in Li, Perrigne, and Vuong (2000). However, we also consider a variation of the model in which the

${ }^{35}$ Appendix A demonstrates that with the model of entry and bidding given in Example 1, our maintained assumptions remain valid in the presence of this sample selection.

${ }^{36}$ As a robustness check, we will also examine the subset of auctions that exclude drainage tracts. 
MMS bid rejection policy is modeled with a random secret reserve price, fit to the data, that responds to tract characteristics and the number of bids received.

Typically a given tract will have eight neighbors, only some (or none) of which will be "active" (under lease). Limited forms of joint bidding were permitted in these auctions. Following the prior literature, we model each bid as coming from a generic "bidder," which might be solo firm or a bidding consortium ${ }^{37}$ Our measure of the number of neighbor firms (distinct owners of leases on neighbor tracts) accounts for the presence of joint bidding by linking together firms that have bid together previously in the same neighborhood, following the criteria developed by AradillasLopez, Haile, Hendricks, and Porter (2017).

Our tract characteristics $X_{t}$, all measured as of the time of the auction, include the number of active neighbor leases, whether the tract is isolated (no active neighbors), the number of firms that bid for neighbor leases, whether the tract was offered previously (attracting no bidders or being relinquished by a prior leaseholder), whether a lease has expired on a neighbor tract, the number of neighbor tracts previously drilled, the number of "hits" on neighbor tracts, average water depth (and its square), and the royalty rate associated with the lease. We present a summary of these auction characteristics in Table 2. Below we will also incorporate year fixed effects.

Following Example 1, we consider one instrument for participation: the number of neighbor firms ${ }^{38}$ This variable is likely to affect bidder entry because ownership of a neighbor tract is likely to reduce the cost of assessing the value of the current tract. As discussed in Appendix $\mathrm{A}$, when we condition on the number of neighbor tracts and the set of firms that previously bid for those tracts, variation in the number of neighbor firms is determined entirely by the realizations of bidder signals at prior

\footnotetext{
${ }^{37}$ Hendricks and Porter (1992) and Hendricks, Porter, and Tan (2008) examine empirical and theoretical aspects of joint bidding in these auctions.

${ }^{38}$ Note that when the number of neighbor firms is zero, the lease is defined as "isolated." The impact of isolation is captured directly through the dummy variable for isolation.
} 
Table 2: Summary Statistics

\begin{tabular}{l|ccc}
\hline \hline Variable & Mean & Median & Std Dev \\
\hline \# active neighbor leases & 1.48 & 0.00 & 1.96 \\
isolated tract & 0.59 & 1.00 & 0.49 \\
\# firms that bid for neighbors & 2.37 & 0.00 & 3.56 \\
re-offered tract & 0.20 & 0.00 & 0.40 \\
neighbor expired & 0.31 & 0.00 & 0.46 \\
\# neighbors drilled & 1.69 & 1.00 & 2.25 \\
\# neighbor hits & 0.65 & 0.00 & 1.30 \\
water depth & 0.20 & 0.14 & 0.28 \\
royalty rate & 16.10 & 16.00 & 1.35 \\
\# neighbor firms & 0.89 & 0.00 & 1.27 \\
estimated ex-post value (millions 1982 dollars) & -6.50 & -6.69 & 46.38 \\
\hline
\end{tabular}

auctions, and therefore independent of $U_{t}$ under our maintained assumptions. Table 2 includes summary statistics for this instrument as well as for an estimate of the realized value of each tract (see, e.g., HPP for details). We do not use these estimated "ex post values" in our analysis, but the table demonstrates the substantial variation in ultimate value of a lease, even among those attracting at least one bid.

\subsection{Baseline Model Estimates}

Here we report estimates of the entry model, index function, and joint distribution of equilibrium bids, using our baseline empirical specification. We specify the entry model as an ordered probit and use a linear index function $\gamma$. We estimate the joint distributions of log homogenized bids using a Bernstein polynomial approximation of order $m=4$ and Gaussian copula ${ }^{39}$ In general we estimate separate joint distributions for each value of $n_{t}$. However, for large values of $n_{t}$ we observe relatively few auctions (recall Table 1), leading us to use a specification in which all auctions with $n_{t} \geq 11$ share the same marginal distribution of homogenized bids and the same

\footnotetext{
${ }^{39}$ Our choice of $m$ reflects our experience with the tradeoff between flexibility an precision using similar sample sizes in our Monte Carlo simulations.
} 
copula correlation parameter. Altogether, this leads to a baseline specification with 151 parameters to be estimated.

\subsubsection{Entry Model}

Table 3 shows our estimates of the entry model parameters. We also report both the standard parametric standard error estimates ("SE"), which ignore spatial dependence, and standard errors obtained from the spatial block bootstrap ("SE (BB)") 40 As discussed in section 2 (see also Appendix A), the function $\eta$ characterizes the effect of $Z_{t}$ on entry but generally will not reveal the (causal) effects of $X_{t}$. Thus one must interpret the estimated coefficients here with caution. Nonetheless, these parameter estimates, particularly those that are statistically distinguishable from zero, generally have intuitive signs. ${ }^{41}$ The coefficient on $Z_{t}$ is positive (consistent with the prediction of our motivating example) and statistically significant, supporting its value in providing a source of variation in bidder entry.

\subsubsection{Index Function and Bid Distribution}

Table 4 displays estimates of the index parameters $\gamma_{x}$ and $\gamma_{u}$, along with their estimated standard errors. The estimates indicate a strong effect of the unobserved heterogeneity on bids. Because $U_{t}$ is normalized to have a uniform distribution, the coefficient implies that a one standard deviation increase in $U_{t}$ drives up bids (and valuations) by roughly $33 \%$. We again caution that the estimated coefficients on $X_{t}$ do not have the usual causal interpretations. Nonetheless, the statistically significant coefficients on $X_{t}$ generally have intuitive signs.

We will not report estimates of the Bernstein polynomial parameters. However,

\footnotetext{
${ }^{40}$ We report results based on 800 bootstrap replications throughout.

${ }^{41}$ Note that, holding the number of neighbor firms fixed, a larger number of active neighbor leases is associated with lower participation in auctions of neighboring tracts and may therefore indicate a less desirable neighborhood.
} 
Table 3: Entry Model Estimates

\begin{tabular}{l|c|ccc}
\hline \hline & & Est. & SE & SE (BB) \\
\hline \# active leases & $\mathrm{X}$ & -0.089 & 0.028 & 0.033 \\
isolated lease & & 0.518 & 0.089 & 0.100 \\
\# firms that bid for neighbors & & 0.016 & 0.011 & 0.012 \\
reoffered tract & & -0.201 & 0.065 & 0.075 \\
neighbor expired & & -0.044 & 0.070 & 0.079 \\
\# neighbors drilled & & -0.046 & 0.028 & 0.030 \\
\# neighbor hits & & 0.076 & 0.028 & 0.027 \\
depth & & -0.558 & 0.179 & 0.283 \\
depth squared & & 0.120 & 0.068 & 0.132 \\
royalty rate & & -0.002 & 0.017 & 0.022 \\
time controls & & Sale & year dummies \\
\# neighbor firms & & & \\
\hline
\end{tabular}

Table 5 shows our estimates of the Gaussian copula correlation parameters $\rho_{n}$. The point estimates are positive, consistent with our assumption of positive dependence between bidders' private information. Although the point estimates generally suggest greater correlation at higher levels of competition, a Wald test fails to reject the hypothesis of equal copula correlation for all values of $n 42$ A Wald test strongly rejects (p-value $<0.001)$ the null that all $\rho_{n}$ are zero. Because homogenized bids are strictly increasing functions of signals, this implies rejection of the hypothesis of independent bidder types. This finding is of some importance on its own. Common knowledge unobservables and correlated private information are two distinct phenomena with different implications for behavior and policy. Often only one of these two sources of correlation between bids has been permitted in applications.

\footnotetext{
${ }^{42}$ While not statistically distinguishable from the others, the estimated correlation parameter for the nine-bidder auctions stands out. A close examination of the data revealed no clear explanation for this. We do not use auctions with more than seven bidders in our tests for common values below, and results are similar when we omit auctions with $n_{t}>7$ from the estimation altogether.
} 
Table 4: Index Function Estimates

\begin{tabular}{l|c|ccc}
\hline \hline & & Est. & SE & SE (BB) \\
\hline \# active leases & $\mathrm{X}$ & 0.016 & 0.018 & 0.021 \\
isolated lease & & 0.042 & 0.059 & 0.087 \\
\# firms that bid for neighbors & & 0.029 & 0.008 & 0.011 \\
reoffered tract & -0.167 & 0.047 & 0.067 \\
neighbor expired & & -0.280 & 0.047 & 0.067 \\
\# neighbors drilled & & 0.049 & 0.019 & 0.024 \\
\# neighbor hits & -0.022 & 0.020 & 0.025 \\
depth & & -0.224 & 0.135 & 0.269 \\
depth squared & & 0.092 & 0.064 & 0.118 \\
royalty rate & & -0.006 & 0.010 & 0.017 \\
time controls & & Sale year dummies \\
unobserved heterogeneity & $\mathrm{U}$ & \multicolumn{3}{|c}{} \\
\hline
\end{tabular}

Table 5: Copula Correlation Estimates

\begin{tabular}{c|ccc}
\hline \hline$n$ & Est. & SE & SE (BB) \\
\hline 2 & 0.044 & 0.041 & 0.050 \\
3 & 0.031 & 0.029 & 0.040 \\
4 & 0.089 & 0.031 & 0.032 \\
5 & 0.136 & 0.030 & 0.041 \\
6 & 0.112 & 0.035 & 0.037 \\
7 & 0.111 & 0.044 & 0.043 \\
8 & 0.133 & 0.039 & 0.040 \\
9 & 0.295 & 0.071 & 0.067 \\
10 & 0.161 & 0.052 & 0.068 \\
$11-18$ & 0.109 & 0.025 & 0.020 \\
\hline
\end{tabular}




\subsection{Decomposition of Correlation and Variance}

Correlation in private information is just one reason bids are correlated within an auction; auction observables and unobservables also play a role. These auction characteristics also contribute to the overall variance of the equilibrium bids. We can use the results above to describe the contributions of each factor.

Table 6 shows a decomposition of within-auction pairwise correlation and overall variance of the log bids 43 The figures presented here reflect calculations using simulated draws from the estimated model. Figures in the first column, labeled "log $B_{i t}^{0}$," are for the homogenized log bids. Here the pairwise correlation reflects the correlation among signals, the nonlinearity of the bidding strategy (and log transformation), and the fact that, all else equal, bid levels vary with the number of competitors in the auction. Similarly, the variance in this column reflects the variability in bidders' assessments of tract values, as well as variation in bidding strategies across auctions with different numbers of entrants.

A natural way to characterize the contributions of unobservables is to examine the correlation and variance arising from variation in $\gamma\left(x, U_{t}\right)$ at a representative value of $x$. With our linear specification of $\gamma\left(X_{t}, U_{t}\right)$, this variation is identical for all $x$. Thus, in the second column, labeled " $\log B_{i t}^{0}+\gamma_{u} U_{t}$," we add the contribution of auction-level unobservables.

Quantifying the contribution of the observables $X_{t}$ can be more problematic, at least when we allow our model to represent an environment in which the underlying unobservables may be correlated with $X_{t}$ (see Appendix A). In that case, variation with $X_{t}$ in the conditional mean $E_{U_{t}}\left[\gamma\left(X_{t}, U_{t}\right) \mid X_{t}\right]$ or in the $u$ th quantile $\gamma\left(X_{t}, u\right)$ would correctly describe variation in bids associated with variation in $X_{t}$, but need not represent the variation caused by variation in $X_{t}$ alone-i.e., that arising when

\footnotetext{
${ }^{43}$ By "within-auction pairwise correlation" we refer to the Pearson correlation coefficient for pairs of bids within the same auction.
} 
Table 6: Decomposition of Log Bid Correlation and Variance

\begin{tabular}{llll}
\hline \hline $\log B_{i t}^{0}$ & $\log B_{i t}^{0}+\gamma_{u} U_{t}$ & $\log B_{i t}^{0}+\gamma_{u} U_{t}+X_{t}^{\prime} \gamma_{x}$ & $\log B_{i t}$ \\
\hline
\end{tabular}

Within-Auction Pairwise Correlation

$\begin{array}{llll}0.133 & 0.210 & 0.222 & 0.425\end{array}$

$\underline{\text { Variance }}$

1.520

1.669

1.693

2.292

the actual unobservables ( $E_{t}$ in Example 1) are held fixed. However, once we know the contributions of the log homogenized bids and the unobservables, we know that all remaining correlation/variance in the log bids arises from the auction observables. Again exploiting our linear specification of the index function, we split the contributions of observables into those of the year fixed effects and those of the auction-level covariates. The third column of the table, labeled " $\log B_{i t}^{0}+\gamma_{u} U_{t}+X_{t}^{\prime} \gamma_{x}$ " adds the variation due to auction-level covariates, while the final column, labeled "log $B_{i t}$," adds the contribution of the year fixed effects to yield the total correlation and variance of the equilibrium bids ${ }_{44}^{44}$

Given the wide time span of our data set and the substantial variation across time in underlying market conditions, it is not surprising that the fixed effects account for a substantial portion of the correlation and variance. More interesting is a comparison of the contributions of the auction-level covariates and the auction-level unobservables. The the estimated contribution of the unobservables is roughly six times as large as that of the observed covariates. This is particularly noteworthy

\footnotetext{
${ }^{44}$ Unlike the rest of the decomposition, the order of these last two steps could matter, due to correlation between the covariates and fixed effects. Here, reversing the order has virtually no effect on the implied contributions to the within-auction correlation; however it increases the contribution of the covariates to the bid variance: the impact of unobservables is then only three (rather than six) times as large as that of the observed covariates.
} 
because we selected covariates $X_{t}$ from an unusually rich set of observables based in part on explanatory power in descriptive analysis of bids. Unobserved heterogeneity could be even more important in applications where only a limited set of covariates is available.

\section{Test For Common Values}

\subsection{Testing Approach}

Using the estimates above, we test the null hypothesis of private values against the alternative of common values, relying on the following additional assumption.

Assumption 12. For all $n=2, \ldots, \bar{n}-1$,

$F_{S, V^{0}}\left(S_{1 t}, \ldots, S_{n t}, V_{1 t}^{0}, \ldots, V_{n t}^{0} \mid N_{t}=n\right)=F_{S, V^{0}}\left(S_{1 t}, \ldots, S_{n t}, V_{1 t}^{0}, \ldots, V_{n t}^{0} \mid N_{t}=n+1\right)$.

This is an assumption that $U_{t}$ is the only source of dependence between the number of bidders at auction $t$ and their valuations/signals. Thus, for example, holding $\left(X_{t}, U_{t}\right)$ fixed, variation in $N_{t}$ is not associated with variation in the valuations or in the precision of signals. Given this condition, HHS showed that the homogenized pivotal expected values $w^{0}\left(S_{i t} ; n\right)$ are unaffected by $n$ in a private values auction but decreasing in $n$ in a common values auction 45 This distinction reflects the winner's curse, which is present in (and only in) common values auctions, and which becomes more severe as the number of competitors increases (all else equal) ${ }_{6}^{46}$ HHS also pointed out that under the maintained assumptions of the affiliated values auction model, $w^{0}\left(S_{i t} ; n\right)$ must be weakly decreasing in $n$. Because violations of this requirement indicate a rejection of at least one of our maintained hypotheses, this allows a

\footnotetext{
${ }^{45}$ See also Athey and Haile (2002).

${ }^{46}$ We use the term "winner's curse" to describe the adverse selection faced by a bidder competing against others with informative signals, not to errors or regret on the part of a bidder.
} 
test of the maintained assumptions, including that of equilibrium bidding ${ }^{47}$

To perform the tests, we first construct estimates of the marginal distributions $F_{w}(\cdot ; n)$ of homogenized pivotal expected values conditional on $N_{t}=n$, following the procedure discussed in section 4. We then subject the estimated distributions to tests of the null hypothesis of equality (private values),

$$
F_{w}(w ; n)=F_{w}(w ; n+1) \quad \forall w, n=2, \ldots, 6,
$$

against the one-sided alterative of first-order stochastic dominance (common values),

$$
F_{w}(w ; n) \leq F_{w}(w ; n+1) \quad \forall w, n=2, \ldots, 6,
$$

with the inequality strict for at least some $n$ and $w$. We limit attention to auctions with at most seven bidders in part to ensure that we have a sample of at least 100 auctions for each value of $n$ considered 48 An additional reason, however, is that growth in the severity of the winner's curse with the level of competition tends to diminish quickly as $n$ grows. Intuitively, once a bidder assumes that $n-1$ others have low signals, learning that one additional signal is low conveys little "bad news" unless $n$ is small. Thus, with common values, pivotal expected values are decreasing and, typically, convex in $n .49$ This intuitive feature leads us to expect any evidence for common values to be clearest when comparing distributions at the lowest values $n$ to those at higher levels of $n$.

\footnotetext{
${ }^{47}$ Another testable implication of the maintained hypotheses is that the inverse homogenized bid functions - i.e., the right-hand-side of the first order conditions $(15)$ - be strictly increasing as functions of the log homogenized bid (see Guerre, Perrigne, and Vuong (2000)). We find no violation of this requirement, even before allowing for sampling error (see Figure 2 below.)

${ }^{48}$ Abusing notation slightly, we let $n$ (without an index) represent the number of bidders in a non-specific auction rather than referring repeatedly to "the number of bidders."

${ }^{49}$ This convexity holds in all examples of symmetric common values auctions we are aware of. An interesting open question is whether additional assumptions are needed to prove this as a general property.
} 
We compare pairs of distributions using the one-sided Cramér-Von Mises type statistic

$$
C V M=\int_{-\infty}^{\infty}\left[\hat{F}_{w}\left(w ; \mathbb{N}_{1}\right)-\hat{F}_{w}\left(w ; \mathbb{N}_{2}\right)\right]_{+}^{2} d w
$$

where $[y]_{+}=y \times 1\{y>0\}$ and $\hat{F}_{w}(w ; \mathbb{N})$ is the estimated distribution of $w^{0}\left(S_{i t} ; N_{t}\right)$ conditional on $N_{t}$ lying in a range of values defined by a set $\mathbb{N}$. We focus primarily on sets $\mathbb{N}$ containing two adjacent values of $n$ ("coarse binning"). This pooling is done to reduce the impact of sampling error. However, by combining $n=2$ and $n=3$, where we expect the largest change in the severity of the winner's curse, this pooling may hide the strongest evidence of common values. Thus, we will also consider singleton sets ("fine binning"). In addition to pairwise tests, we will construct a single test statistic for the full range $n=2, \ldots, 7$, based on the maximum statistic (or smoothed maximum) over the pairwise statistics:

$$
C V M^{\max }=\max _{j} \int_{-\infty}^{\infty}\left[\hat{F}_{w}\left(w ; \mathbb{N}_{j}\right)-\hat{F}_{w}\left(w ; \mathbb{N}_{j+1}\right)\right]_{+}^{2} d w
$$

Below we report results for the pairwise and "max" tests for coarse binning, as well as the for the max test for fine binning ${ }^{50}$ We construct p-values for the test statistics using the distribution of (re-centered) test statistics from the spatial block bootstrap procedure described above.

\subsection{Testing Results}

In Figure 1a we show the estimated CDFs under coarse binning, where we compare "low" ( $n \in\{2,3\})$, "medium" $((n \in\{4,5\})$, and "high" $(n \in\{6,7\})$ levels of compe-

\footnotetext{
${ }^{50}$ Appendix D provides the complete test results for the fine binning, including those for the alternative specifications below. In general, these results confirm that, while the fine binning tests are noisier, the strongest evidence for common values comes from comparisons of $n=2$ to $n=3$, where we have relatively large numbers of bidders and where we consistently obtain p-values smaller than those for the max test reported in the text.
} 
tition. Under the null, these distributions should differ only due to sampling error, whereas the alternative of common values implies that the CDFs will shift "northwest" as $n$ increases. The estimated distributions shown here exhibit the stochastic ordering implied by the common values model. Further, as expected, the gap between the distributions for low and medium $n$ is substantially larger than that between the distributions for medium and high $n$. We contrast this with Figure 1b, which shows the estimated distributions obtained when we estimate the model without allowing for unobserved heterogeneity. Here the results suggest stochastic ordering in the direction opposite that predicted by common values. This suggests a misspecified model.

Table 7 shows the p-values obtained from the formal tests. These results confirm what was suggested by the figures above. First consider the comparison between low $n$ and medium $n$ when we allow unobserved heterogeneity ("With UH"). The test for common values implies rejection of private values in favor of common values, with a p-value of 0.021. The smaller gap between the estimated CDFs for medium and high $n$ observed in Figure 1 cannot be statistically distinguished. However, the max tests - for both coarse and fine binning - also imply rejection at significance levels around 2 percent. Consistent with Figure 1, the specification test yields no evidence suggesting misspecification in the model with unobserved heterogeneity.

Contrast these results with those obtained in the model without unobserved heterogeneity ("No UH"). Here, the conclusions are essentially reversed. Not only is there no evidence of common values, but the specification tests suggest misspecification, with all three coarse binning tests yielding p-values below 0.10 . Thus, at least in our data, failing to account for unobserved heterogeneity prevents detection of common values and can erroneously suggest non-equilibrium bidding. This is intuitive, but not a necessary implication of ignoring unobserved heterogeneity. On one hand, when we ignore unobserved heterogeneity and endogenous entry, an "endogenous treatment" bias works against the winner's curse effect we are seeking to detect: auctions with 
Figure 1: Test for Common Values, Baseline Specification

(a) With Unobserved Heterogeneity

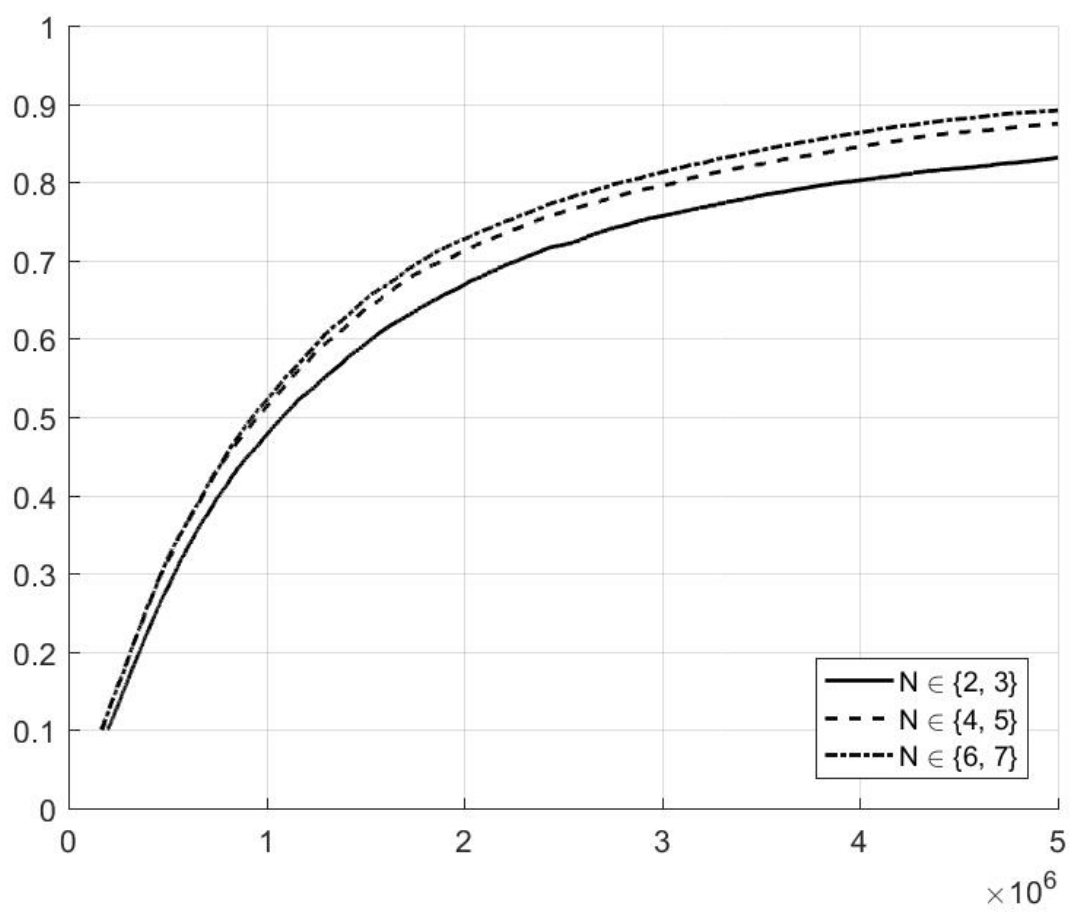

(b) Without Unobserved Heterogeneity

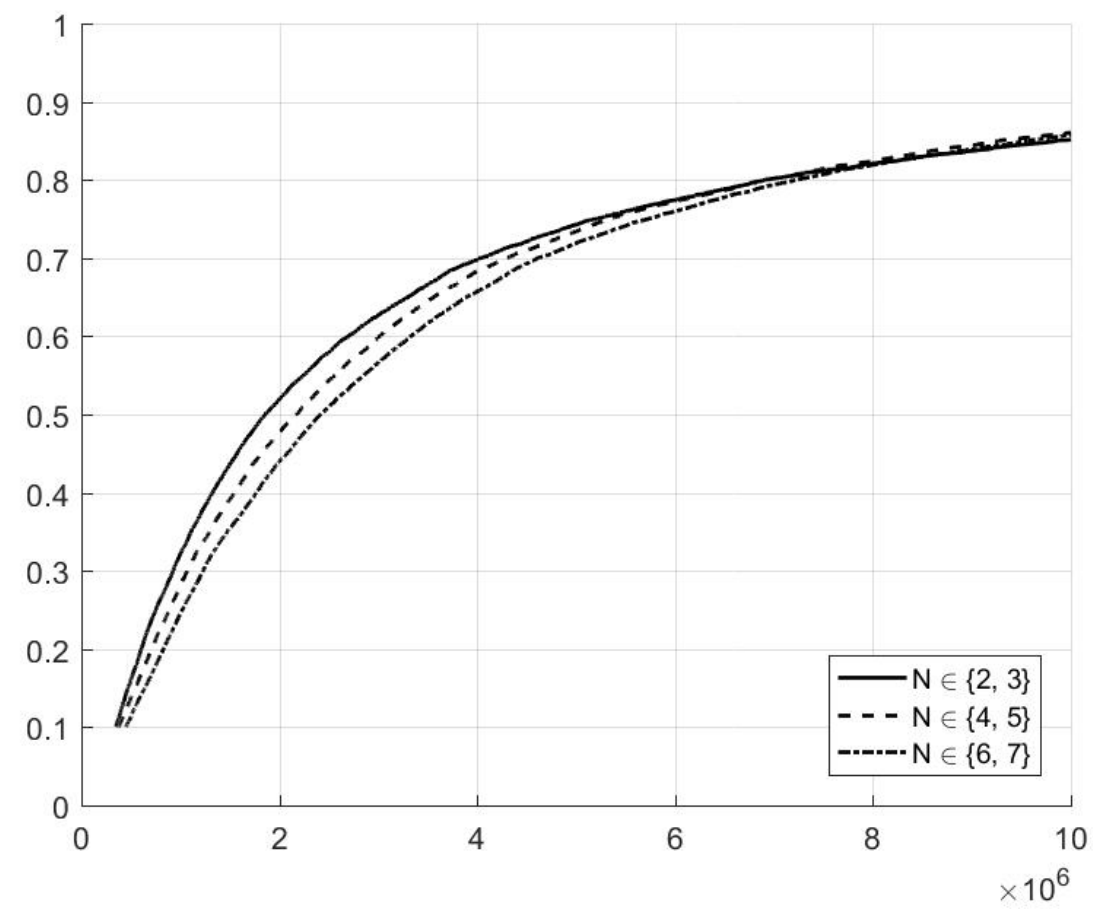


Table 7: Test p-values

Baseline Specification

\begin{tabular}{l|c|c}
\hline \hline \multicolumn{3}{c}{ Test for Common Values } \\
& With UH & No UH \\
\hline$\{2,3\}$ vs. $\{4,5\}$ & 0.021 & 0.299 \\
$\{4,5\}$ vs. $\{6,7\}$ & 0.274 & 0.754 \\
Max (coarse binning) & 0.022 & 0.590 \\
Max (fine binning) & 0.019 & 0.541
\end{tabular}

$\underline{\text { Specification Test }}$

\begin{tabular}{l|c|c} 
& With UH & No UH \\
\hline$\{2,3\}$ vs. $\{4,5\}$ & 0.906 & 0.066 \\
$\{4,5\}$ vs. $\{6,7\}$ & 0.901 & 0.076 \\
Max (coarse binning) & 0.985 & 0.081 \\
Max (fine binning) & 0.922 & 0.239 \\
\hline
\end{tabular}

more bidders may have a larger winner's curse, but they also have more favorable unobservables. So the true effects of $n$ on pivotal expected values could be masked or even reversed. But this intuition is incomplete. The model is misspecified when unobserved heterogeneity is present but ignored in the first-order conditions used to interpret the data. The cumulative distributions recovered in that case are not those of bidders' pivotal expected values. Moreover, the direction of the misspecification bias is unclear, and this bias may vary with $n$. Nonetheless, the results indicate that ignoring unobserved heterogeneity obscures the presence of common values in our sample.

\subsection{Competition, Market Power, and Revenue}

The presence of affiliation and common values can have important implications for auction design (see, e.g., Milgrom and Weber (1982)). For example, this supports the use of royalty payments but suggests that, absent concerns about susceptibil- 
ity to collusion (e.g., Athey, Levin, and Seira (2011)), an ascending auction could produce more revenue. Affiliation and common values also interact with the usual effects of competition. A well known feature of common value auctions is that added bidder competition can lead, counterintuively, to less aggressive bidding, and even to reduced seller revenue 51 The winner's curse is a key force behind this possibility, although Pinkse and Tan (2005) demonstrated that bids can decline in the level of competition due to affiliation of signals alone. Here we use our estimates to examine the equilibrium effects of bidder competition on bid shading, the level of bids, and seller revenue.

In Figure 2 we plot, for different values of $n$, bidders' homogenized pivotal expected values against the associated homogenized bids implied by the first-order condition (10). Recall that pivotal expected values are strictly increasing in bidder types (signals). We know from theory that all types above the lowest possible type shade their bids below their pivotal expected values, and that the degree of bid shading is increasing in type. Here we see that the estimated magnitude of this bid shading is substantial - all curves lie well below the 45 degree line. However, the gap shrinks as the level of competition rises from $n=2$ to $n=7$.

In Figure 3 we plot the estimated homogenized equilibrium bidding strategies $\beta^{0}(\cdot ; n)$, where we normalize bidder signals to lie on $[0,1]$ without loss. These estimated bid functions are strictly increasing as implied by the model (but not imposed). They are also generally increasing with $n$, although this monotonicity reverses at $n=7$. Such a reversal is consistent with the folk wisdom that equilibrium bids "eventually" decrease in the number of competitors in a common values auction (see, e.g., Laffont (1997)). However, to assess whether this reflects more than sampling error, we subject these bid functions to the same type of tests we applied to the pivotal

\footnotetext{
${ }^{51}$ Laffont (1997) reviews the theoretical and empirical literature on this point. See also Hong and Shum (2002).
} 
Figure 2: Competition and Bid Shading

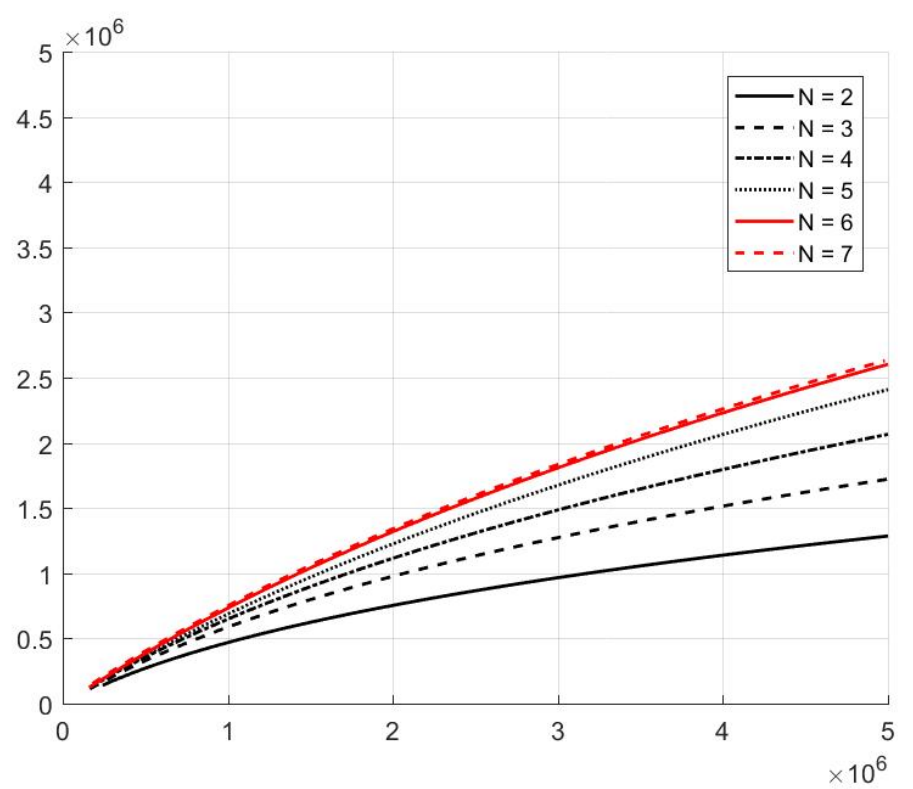

Estimated pivotal expected values on horizontal axis with the associated equilibrium bids on the vertical axis, both in 1982 dollars.

Figure 3: Competition and Equilibrium Bidding Strategies

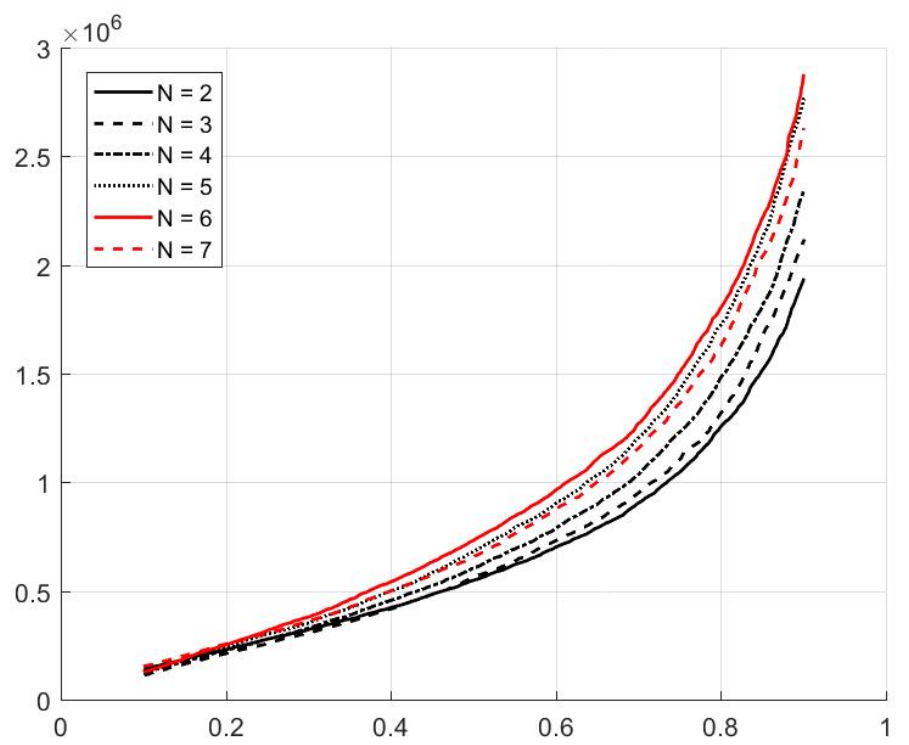

Bidder signals (normalized to $[0,1]$ on the horizontal axis with the associated equilibrium bids (in 1982 dollars) on the vertical axis. 
expected values to test for common values above. Here we test the null hypothesis that the bidding strategies $\beta^{0}(\cdot ; n)$ are increasing in $n$. The test comparing $n=6$ to $n=7$ yields the smallest p-value, which is 0.162 . Thus, we are unable to reject the null at standard significance levels.

Finally, Table 8 shows, at each value of $n$, the implications of our estimates for a seller's expected revenue and median revenue at a homogenized auction (where $X_{t}$ and $U_{t}$ are set to zero). Both measures of revenue increase monotonically with the number of bidders. Notably, even though our point estimates (Figure 3) suggested non-monotonicity with respect to $n$, this effect is overcome by the fact that the winning bid is the maximum among $n$ bids. Thus, even our point estimates give no indication that a seller would profit from restricting entry to reduce the severity of the winner's curse faced by bidders.

Table 8: Competition and Revenues

\begin{tabular}{c|c|c}
\hline \hline $\begin{array}{c}\text { Number of } \\
\text { Bidders }\end{array}$ & $\begin{array}{c}\text { Expected } \\
\text { Revenue }\end{array}$ & $\begin{array}{c}\text { Median } \\
\text { Revenue }\end{array}$ \\
\hline 2 & 1.487 & 0.912 \\
3 & 1.952 & 1.332 \\
4 & 2.431 & 1.679 \\
5 & 3.170 & 2.202 \\
6 & 3.581 & 2.563 \\
7 & 3.591 & 2.565 \\
\hline
\end{tabular}

Revenue in millions of 1982 dollars, for a hypothetical auction with $\gamma\left(x_{t}, u_{t}\right)=0$. 


\section{Alternative Specifications}

Here we consider several alternative specifications, focusing on the tests for common values and specification tests.

\subsection{Random Reserve Price}

Our baseline model ignores the fact that the MMS retained (and sometimes exercised) a right to reject all bids. A bidder who anticipates this rejection possibility would have an incentive for more aggressive bidding than is implied by the standard equilibrium first-order condition. In our sample this effect is likely to be small in auctions attracting at least three bidders, where well over 99 percent of all winning bids were accepted $\sqrt[52]{52}$ However, the threat of rejection could be an important factor in auctions attracting only one or two bidders 53

To incorporate this feature, we follow Hendricks, Porter, and Spady (1989) and Hendricks, Porter, and Wilson (1994)) by modeling bid rejection with a random reserve price whose value is unknown to bidders. From a bidder's perspective, the random reserve price is effectively an additional bid (submitted by the auctioneer), leading to a first-order condition similar to that in the baseline case. We follow Bajari and Hortaçsu (2003) by modeling the reserve price as drawn from the same marginal distribution as a bid, although we rescale the seller's "bid" to fit the observed MMS rejection decisions. We give additional detail and discussion in Appendix C.

Figures $4 \mathrm{a}$ and $4 \mathrm{~b}$ show the estimated marginal distributions of pivotal expected values obtained with this alternative model. These are very similar to the estimates

\footnotetext{
${ }^{52}$ Rejection of the high bid occurred more often at auctions dropped from our sample due either to missing values or to their being auctions of leases on partial-block tracts.

${ }^{53}$ Accounting for this threat enables us to rationalize observed bids in one-bidder auctions as interior solutions obeying a first-order condition and inheriting the index structure of the valuations. Therefore, given the large number of one-bidder auctions in our sample, we include one-bidder auctions in the quasi-maximum likelihood estimation of the model parameters for all specifications.
} 
obtained in the baseline model. In Figure 4a, where we allow unobserved heterogeneity, we see the pattern of first-order stochastic dominance predicted by common values, with the magnitude of the estimated shifts in the distributions declining with $n$. Figure $4 \mathrm{~b}$ again shows that, if unobserved heterogeneity is ignored, we obtain estimated distributions ordered in the direction opposite that implied by common values. As shown in Table 9, the formal tests are also very similar to those in the baseline case 54 In particular, when we account for unobserved heterogeneity, the test for common values leads us to reject private values, with p-values between 0.02 and 0.03. However, we se no evidence of common values when we ignore unobserved heterogeneity.

Table 9: Test p-values Random Reserve Specification

\begin{tabular}{l|c|c}
\hline \hline \multicolumn{2}{c}{ Test for Common Values } \\
& With UH & No UH \\
\hline$\{2,3\}$ vs. $\{4,5\}$ & 0.023 & 0.306 \\
$\{4,5\}$ vs. $\{6,7\}$ & 0.278 & 0.759 \\
Max (coarse binning) & 0.027 & 0.605 \\
Max (fine binning) & 0.028 & 0.664
\end{tabular}

\section{Specification Test*}

\begin{tabular}{l|c|c} 
& With UH & No UH \\
\hline$\{2,3\}$ vs. $\{4,5\}$ & 0.847 & 0.036 \\
$\{4,5\}$ vs. $\{6,7\}$ & 0.839 & 0.071 \\
Max (coarse binning) & 0.960 & 0.075 \\
Max (fine binning) & 0.910 & 0.245 \\
\hline
\end{tabular}

* See footnote 54 and Appendix $\mathrm{C}$.

\footnotetext{
${ }^{54}$ As discussed in Appendix C, with the random reserve price, violations of weak stochastic ordering need not imply rejection of the model. Thus, although the table shows the results of the specification tests, these do not have the usual interpretation.
} 
Figure 4: Random Reserve

(a) With UH

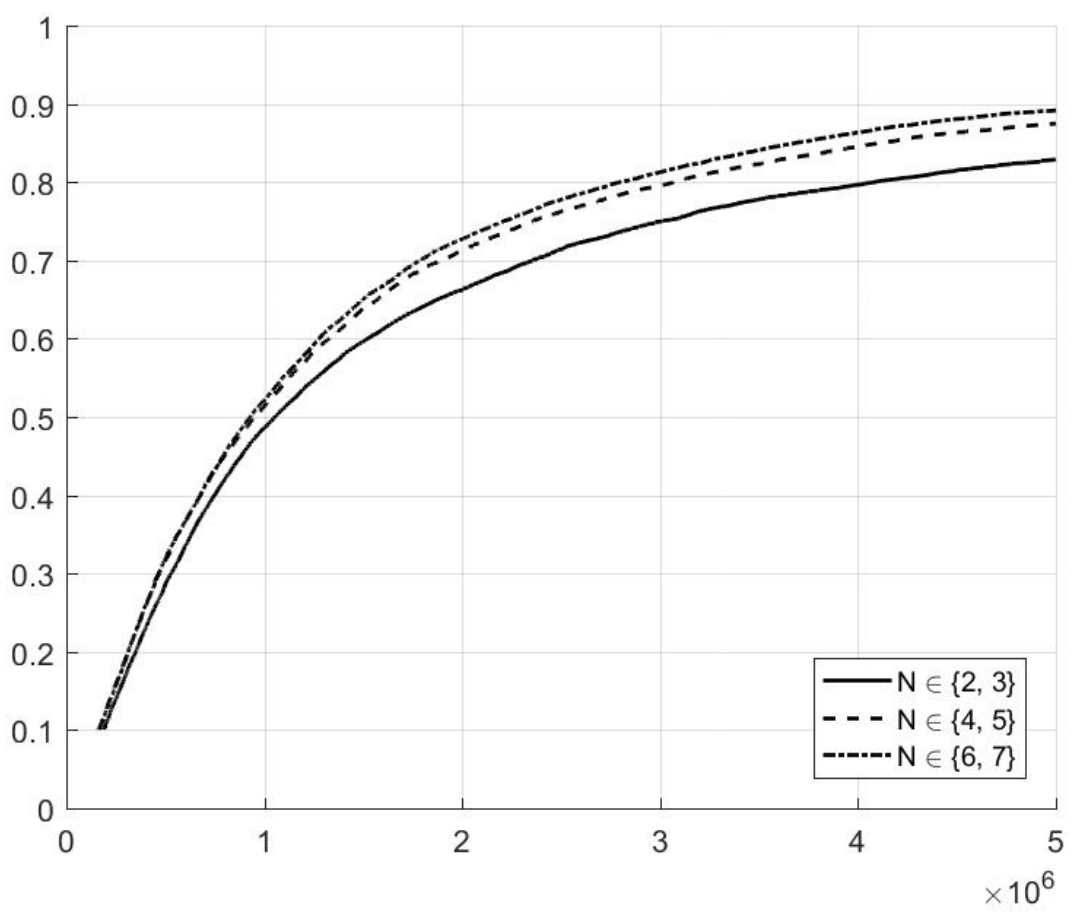

(b) No UH

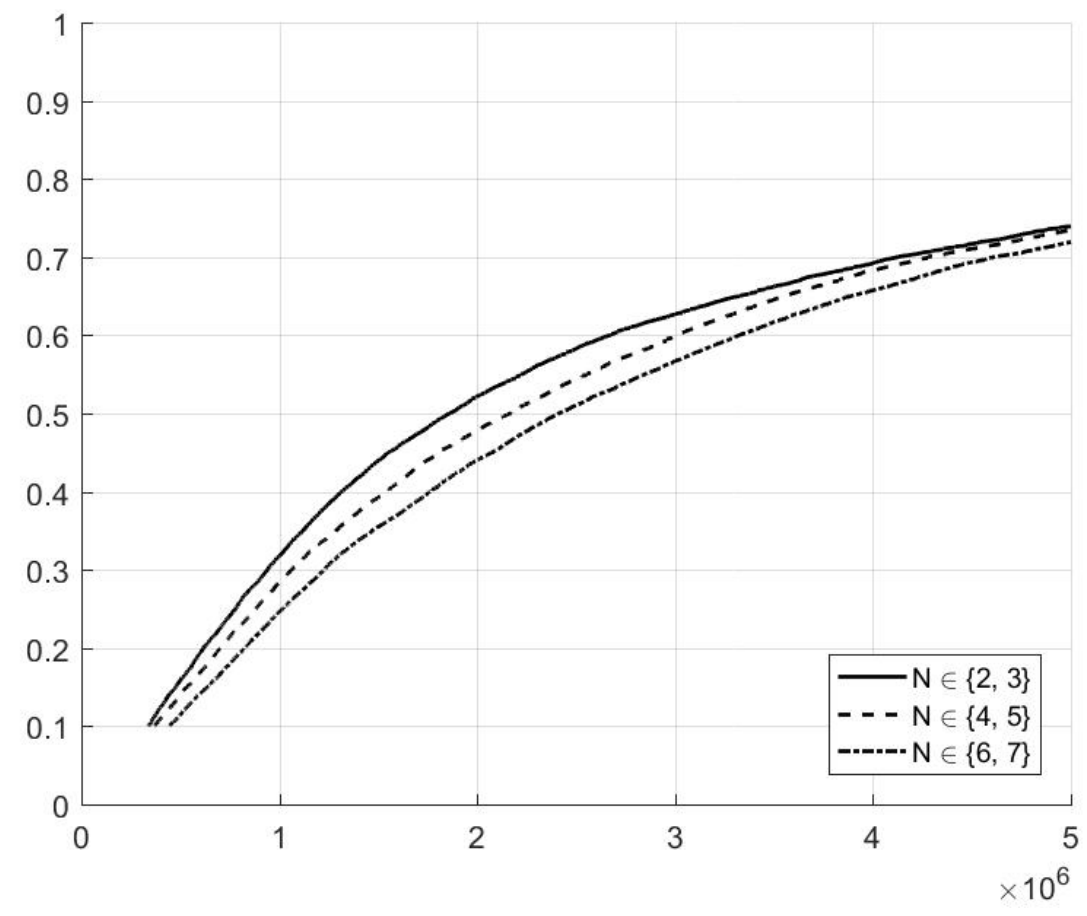




\subsection{Semi-nonparametric Entry Model}

In the results presented so far, the entry model was estimated as an ordered probit: in the notation of section 4.1.1, the distribution $H(\cdot)$ was specified as the standard normal. Although $H$ could be chosen arbitrarily if we specified each $\alpha_{n}(x, z)$ nonparametrically, with our parametric specification of the thresholds $\alpha_{n}(x, z)$ the choice of $H$ could matter. Thus, we consider here an alternative semi-nonparametric specification in which $H(\cdot)$ is approximated flexibly with Hermite polynomials.

Following Gallant and Nychka (1987), $H$ is specified as the continuous distribution function with density

$$
h(\epsilon)=\frac{1}{\theta_{h}}\left(\sum_{k=0}^{\tilde{K}} \sigma_{k} \epsilon^{k}\right)^{2} \phi(\epsilon),
$$

where $\tilde{K}$ is the order of the Hermite polynomial approximation, $\sigma_{k}$ are parameters, $\phi$ is the standard normal density, and $\theta_{h}=\int_{-\infty}^{\infty}\left(\sum_{k=0}^{\tilde{K}} \sigma_{k} \epsilon^{k}\right)^{2} \phi(\epsilon) d \epsilon$. We set $\tilde{K}=3$.

Because the entry model affects only the way that we control for unobserved heterogeneity, we examine here only the results obtained from the model allowing unobserved heterogeneity. In Figure 5 we plot the estimated distributions of homogenized pivotal expected values obtained when we use this alternative specification of the entry model. These distributions exhibit patterns very similar to those from the baseline model. Table 10 confirms that the formal test results are also very similar. Indeed, the statistical evidence of common values is slightly stronger (smaller p-values) with this more flexible specification. 
Figure 5: SNP Entry Model

With UH

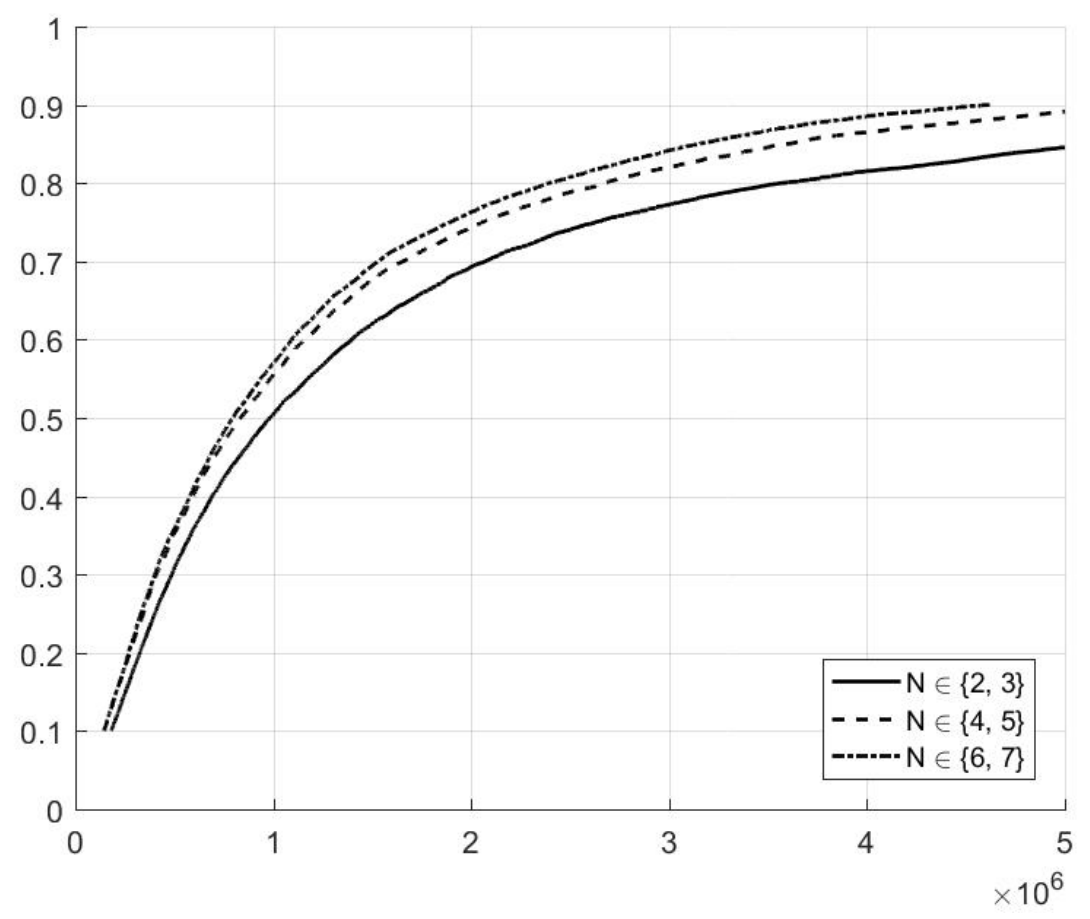


Table 10: Test p-values

With SNP Entry Model

\begin{tabular}{l|c}
\hline \hline \multicolumn{2}{c}{ Test for Common Values } \\
& With UH \\
\hline$\{2,3\}$ vs. $\{4,5\}$ & 0.014 \\
$\{4,5\}$ vs. $\{6,7\}$ & 0.198 \\
Max (coarse binning) & 0.015 \\
Max (fine binning) & 0.004
\end{tabular}

$\underline{\text { Specification Test }}$

\begin{tabular}{l|c} 
& With UH \\
\hline$\{2,3\}$ vs. $\{4,5\}$ & 0.923 \\
$\{4,5\}$ vs. $\{6,7\}$ & 0.948 \\
Max (coarse binning) & 0.995 \\
Max (fine binning) & 0.933 \\
\hline
\end{tabular}

\subsection{No Year Fixed Effects}

Our previous specifications included year fixed effects. These provide flexible control for a number of common-knowledge time-varying factors such as macro shocks, variation in oil and gas prices, changes in industry structure, regulatory changes, etc., which vary substantially over the three decades of our sample. We saw the importance of this temporal variation in Table 6. However, in some applications one might not have sufficient sample size to allow such flexibility. Without the fixed effects, time varying factors would be an additional source of unmeasured heterogeneity, and it is interesting to explore what happens in such cases. We therefore consider a specification that drops the year fixed effects.

The estimated distributions of pivotal expected values are shown in Figures 6 a and 6b. When we account for unobserved heterogeneity and endogenous entry we obtain estimated distributions of pivotal expected values that are again ordered as we would 
expect under common values. The specification ignoring unobserved heterogeneity yields estimated distributions that are ordered - now more sharply than in the baseline specification - in the direction indicating misspecification of the model.

When we examine the formal test results in Table 11, the statistical evidence aligns with the impression given by the figures. For the model allowing unobserved heterogeneity, the rejections of private values in favor of common values are at slightly larger significance levels than in the baseline specification, particularly in the case of fine binning. However, the more striking difference is the stronger rejection of the model that assumes no unobserved heterogeneity. This is as we would expect: as more auction-level factors are forced into the unobservable, the misspecification implied by ignoring unobserved heterogeneity is likely to be more severe.

Table 11: Test p-values

No Year Fixed Effects

\begin{tabular}{l|c|c}
\hline \hline \multicolumn{3}{c}{ Test for Common Values } \\
& With UH & No UH \\
\hline$\{2,3\}$ vs. $\{4,5\}$ & 0.024 & 0.406 \\
$\{4,5\}$ vs. $\{6,7\}$ & 0.319 & 0.841 \\
Max (coarse binning) & 0.028 & 0.748 \\
Max (fine binning) & 0.095 & 0.985
\end{tabular}

$\underline{\text { Specification Test }}$

\begin{tabular}{l|c|c} 
& With UH & No UH \\
\hline$\{2,3\}$ vs. $\{4,5\}$ & 0.976 & 0.069 \\
$\{4,5\}$ vs. $\{6,7\}$ & 0.744 & 0.009 \\
Max (coarse binning) & 0.937 & 0.009 \\
Max (fine binning) & 0.942 & 0.625 \\
\hline
\end{tabular}


Figure 6: No Year Fixed Effects

(a) With UH

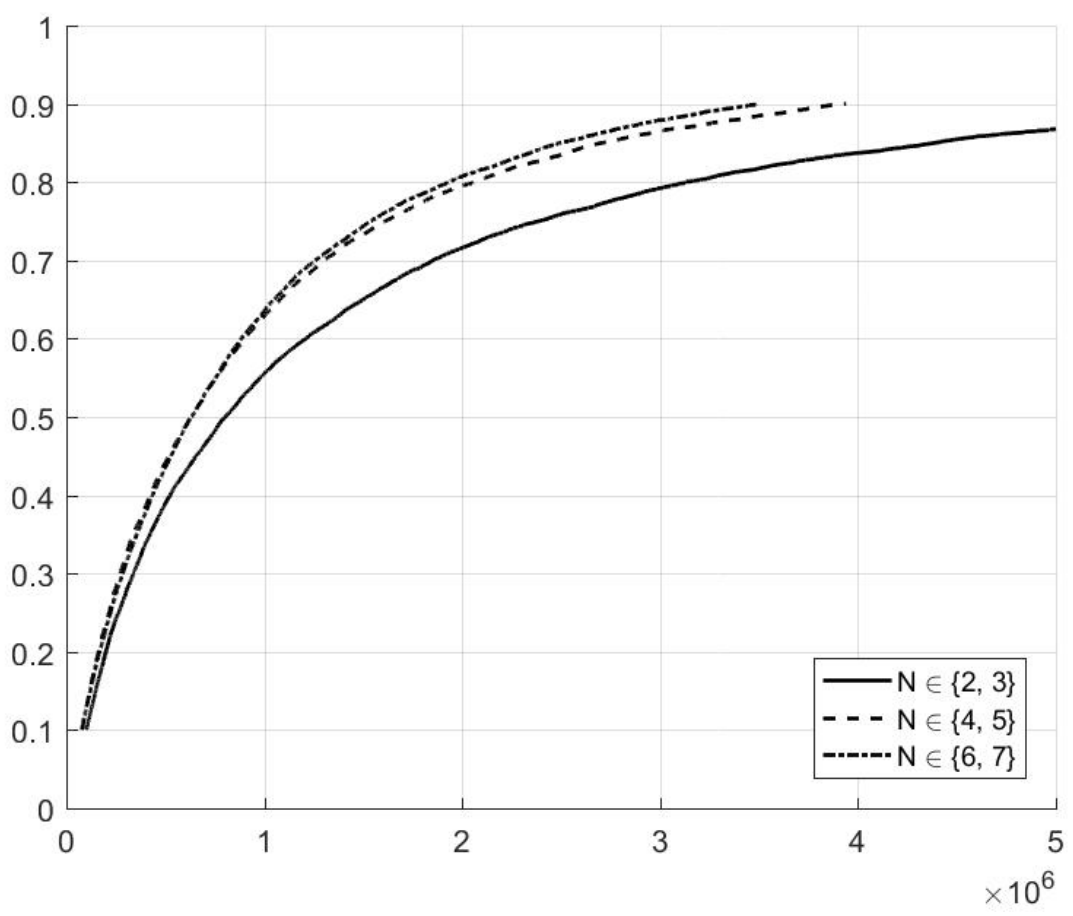

(b) No UH

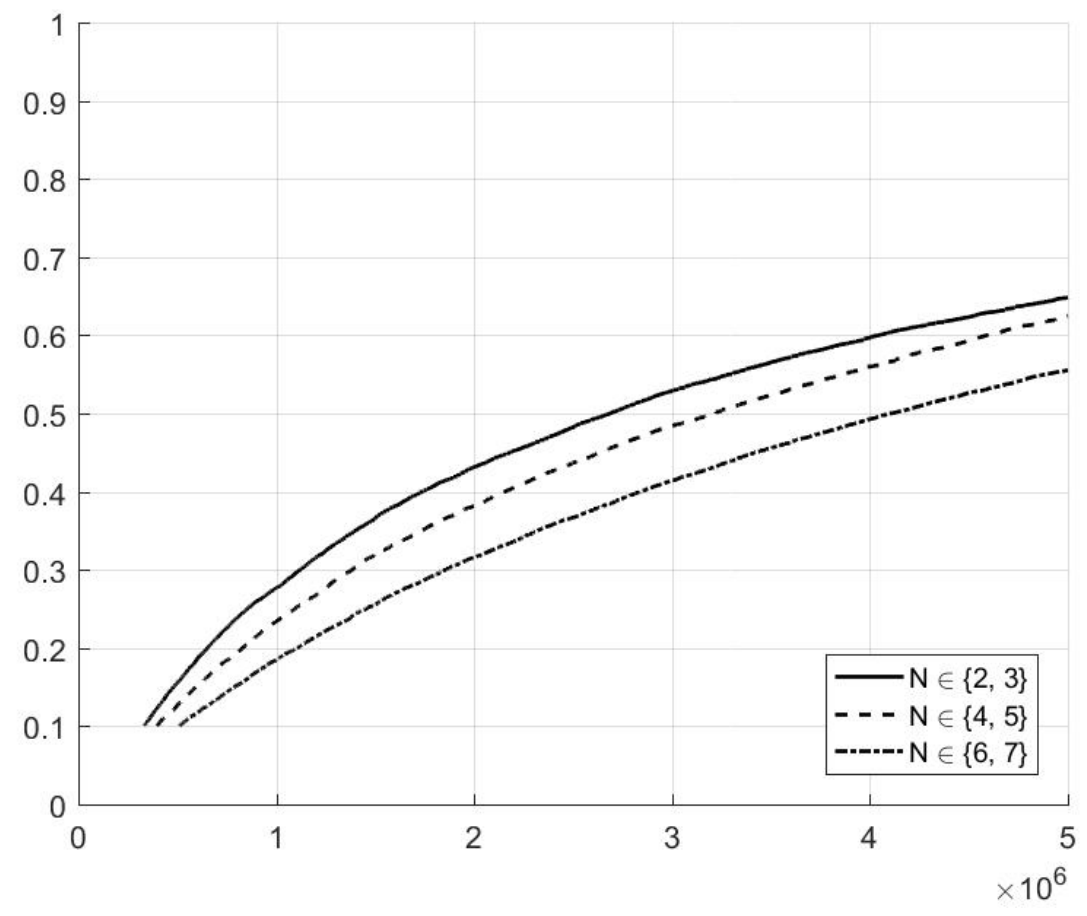




\subsection{No Drainage Tracts}

"Drainage" tracts are those adjacent to productive leases. It is natural to imagine that owners of neighbor leases have an advantage in assessing the value of adjacent tracts. The path-breaking paper of Hendricks and Porter (1988) focused on drainage tracts and assumed that only the neighbor firm had access to a private signal of the tract value, whereas non-neighbors relied solely on common knowledge information. Our model of entry in OCS auctions (see Example 1) can be viewed as relaxing this structure by allowing less extreme forms of asymmetries in signal acquisition costs and permitting competition between multiple neighbors 55 However, our model is one in which bidders choosing to acquire signals have private information of (ex ante) equal precision: bidders are symmetric in the auction stage. This is consistent with the fact that the frequency of winning conditional on bidding and on the number of bidders is virtually identical for neighbors and non-neighbors in our sample. However, if there is significant private information that can be obtained only by drilling, this is something not permitted by our model. We therefore repeat the analysis dropping all drainage tracts.

Unsurprisingly, restricting the sample in this way eliminates much of the variation in our instrument. This is an important limitation. Recalling the discussion in section 3, it means that the results in this case will be more heavily reliant on the functional form of the index function $\gamma$, and are likely to be less precisely estimated. The estimated distributions in this case, shown in Figures $7 \mathrm{a}$ and $7 \mathrm{~b}$, are in fact very similar to those obtained from the full sample. However, the formal test results show that there is indeed a loss of precision. When we allow for unobserved heterogeneity, the coarse binning test for common values comparing "low" and "medium" competition

\footnotetext{
${ }^{55}$ Hendricks and Porter (1988) assume that if there are multiple neighbors, only one submits a serious bid. This leads to the result that non-neighbors bid in equilibrium despite having no information, using mixed strategies. Such behavior is possible in equilibrium when there is no cost of bidder entry.
} 
yields a p-value of 0.162 , with a p-value of 0.168 for the max test. With fine binning, the max test yields a p-value of 0.075 . The fine binning comparison between $n=2$ and $n=3$, where we expect the largest effect of the winner's curse, yields a p-value of 0.030. Specification tests applied to the model ignoring unobserved heterogeneity fail to reject at conventional significance levels, with p-values between 0.13 and 0.19 from the coarse binning tests.

Thus, while we can still reject private values in favor of common values (at the $10 \%$ level or $5 \%$ level using one of the fine binning tests), the statistical evidence is less definitive. These results indicate that, while the patterns in the estimated distributions obtained from the full sample are robust to exclusion of the drainage tracts, the variation in the instrument these tracts provide is important for the precision of the results.

Table 12: Test p-values

No-drainage Specification

$\underline{\text { Test for Common Values }}$

\begin{tabular}{l|c|c} 
& With UH & No UH \\
\hline$\{2,3\}$ vs. $\{4,5\}$ & 0.162 & 0.344 \\
$\{4,5\}$ vs. $\{6,7\}$ & 0.485 & 0.651 \\
Max (coarse binning) & 0.168 & 0.463 \\
Max (fine binning) & 0.075 & 0.508
\end{tabular}

Specification Test

\begin{tabular}{l|c|c} 
& With UH & No UH \\
\hline$\{2,3\}$ vs. $\{4,5\}$ & 0.856 & 0.188 \\
$\{4,5\}$ vs. $\{6,7\}$ & 0.742 & 0.134 \\
Max (coarse binning) & 0.863 & 0.149 \\
Max (fine binning) & 0.724 & 0.246 \\
\hline
\end{tabular}


Figure 7: No Drainage Tracts

(a) With UH

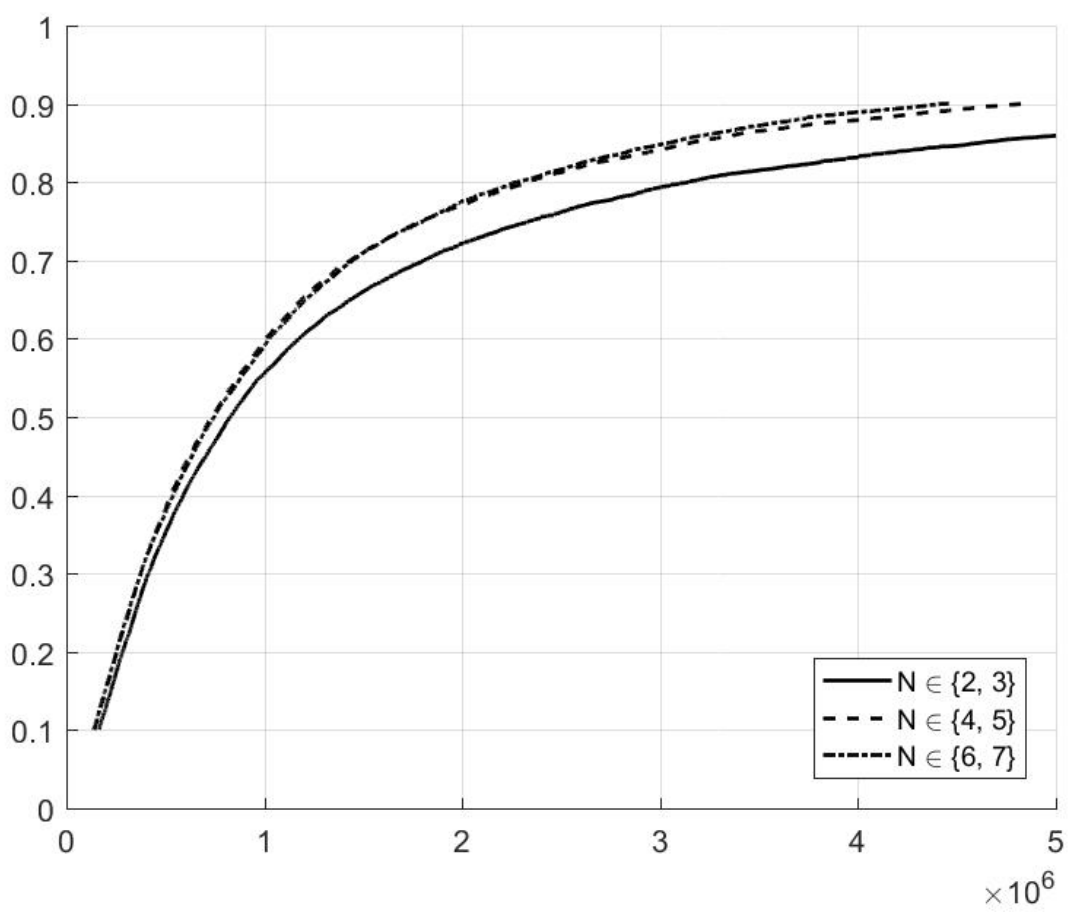

(b) No UH

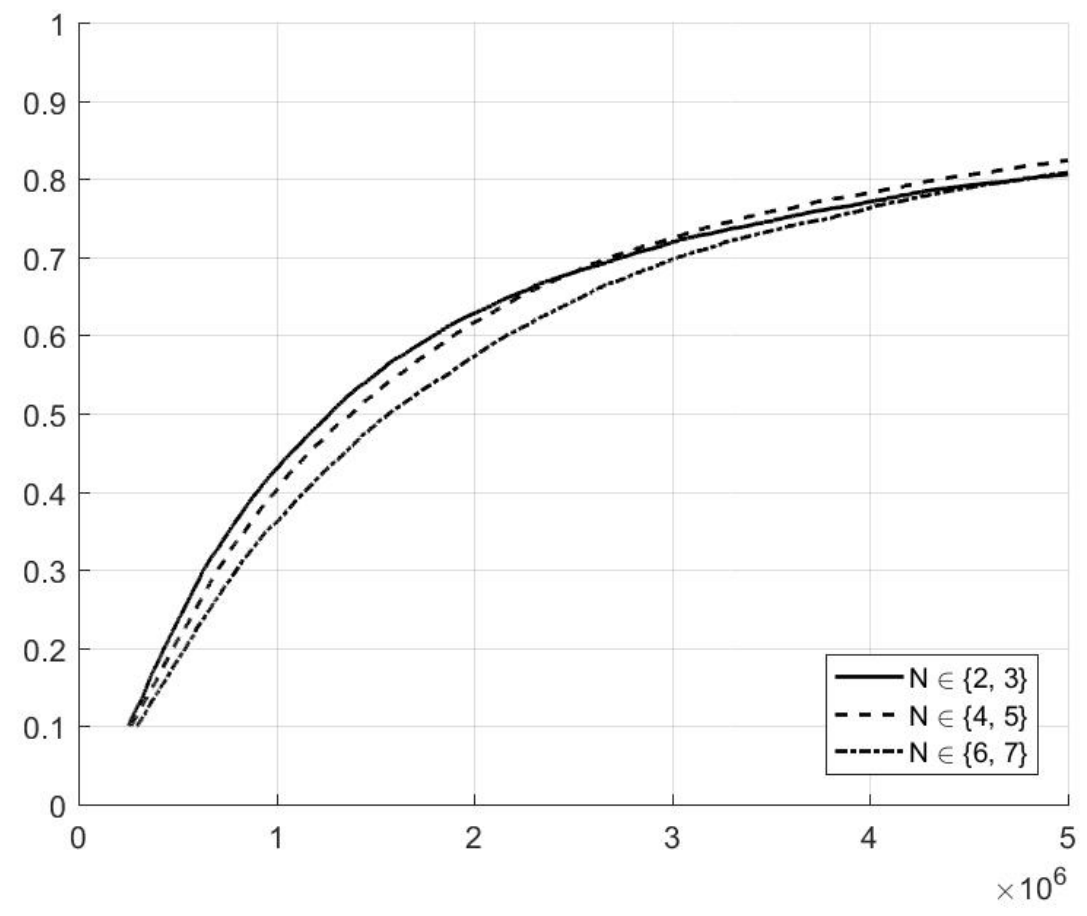




\section{Conclusion}

We have proposed an empirical approach to first-price sealed bid auctions with affiliated values, unobserved auction-level heterogeneity, and endogenous bidder entry. Although our approach offers several conceptual and practical advantages for our empirical study of OCS auctions, it relies on several important assumptions that will be more suitable in some applications than others. Further work may identify ways to relax some of our assumptions or trade them for others. All econometric methods allowing unobserved heterogeneity in first-price auctions require compromises, and in practice the best approach will vary with the application and questions at hand.

Our analysis of the OCS auction data leads us to reject the private values model in favor common values, a conclusion that is robust across a variety of specifications. We found that ignoring unobserved heterogeneity can hide the presence of common values, due in part to the fact that positive dependence between bidder entry and unobserved tract value works against the effects that exogenous changes in the number of bidders have on the severity of the winner's curse. However, in practice our specification tests allow us to reject specifications that ignore unobserved heterogeneity.

While our empirical results confirm the conventional wisdom that oil lease auctions should be viewed as common values auctions, they also raise challenges. We obtained a useful form of partial identification for a common values model, but common values models - even without unobserved heterogeneity - generally are not point identified without assumptions and data beyond what we required. Thus it will be important to continue pursuit of approaches to identification that exploit the features of particular settings (see, e.g., HPP or Somaini (2015)) and to explore extensions permitting unobserved heterogeneity. It may also prove productive to pursue other forms of partial identification that can be used to address positive and normative questions. The recent work of Syrgkanis, Tamer, and Ziani (2018) provides one such approach. 


\section{Appendices}

\section{A Equilibrium Entry in a Model of OCS Auctions}

Here we consider a particular extensive form game of entry and bidding that is motivated by our application and yields the reduced form (3) for the entry outcome presented in the text, including the assumed weak monotonicity conditions.56 This example also demonstrates how our model can accommodate auction-specific unobservables that are of arbitrary dimension and correlated with auction-specific observables, despite the apparent contradiction to our assumption that $U_{t}$ is a scalar and independent of $X_{t}$. Accommodation of such correlation requires that we allow the interpretation of $U_{t}$ to vary with the vector $X_{t}$. This precludes identification of (causal) effects of covariates on the auction; but in typical auction applications auction-level observables are primarily confounding factors to be controlled for rather than factors whose effects are of direct interest themselves. This section also motivates the instrument used in our application. Finally, we discuss here the selection on unobservables that could be implied by considering only auctions attracting at least one bid, as necessitated by our data. We demonstrate that such selection introduces only an additional way in which the interpretation of $U_{t}$ varies with $X_{t}$.

\section{A.1 Model}

Consider a game of entry and bidding for the lease of a tract $t$. Let $\mathcal{I}$ denote the set of all potential bidders ("firms"), and let $I=|\mathcal{I}|$. The set $\mathcal{I}$ can be partitioned into the set $\mathcal{Z}_{t}$ of "neighbor firms" - holders of active leases on adjacent ("neighbor") tracts - and all other firms, $\mathcal{I} \backslash \mathcal{Z}_{t}$. Denote the number of neighbor firms by $Z_{t}=\left|\mathcal{Z}_{t}\right|$.

\footnotetext{
${ }^{56}$ Example 1 in the text provided a sketch.
} 
Let $V_{i t}$ denote the value of the lease to firm $i$ ( $i$ 's "valuation"). Let $X_{t}$ and $E_{t}$ denote, respectively, observed and unobserved (to us) characteristics of lease $t$ that affect bidders' valuations. Let $X_{t}$ include (among other relevant characteristics) the number of active leases on neighboring tracts and the set of bidders for each of those leases ${ }^{57}$ We make no restriction on the dimension of $E_{t}$ and do not require independence between $X_{t}$ and $E_{t}$.

The game consists of two stages. In the second stage, lease $t$ is offered by firstprice auction to the $N_{t}$ bidders who enter in the first stage. We assume there is no binding reserve price in the auction 58 In the first stage, bidders simultaneously choose whether to incur an entry cost in order to acquire a signal and participate in the auction 59 Let $c_{i}\left(x_{t}\right)$ denote the entry cost for firm $i$. Neighbors have lower entry costs. In particular, $c_{i}\left(x_{t}\right)=c\left(x_{t}\right)$ for a neighbor firm, whereas non-neighbor firms have entry $\operatorname{costs} c_{i}\left(x_{t}\right)=c\left(x_{t}\right)+\delta\left(x_{t}\right)$, with $\delta\left(x_{t}\right)>0$.

Firms acquiring signals become "bidders" and learn the number of competitors they face. Let $N_{t}$ denote the number of bidders. Let $S_{i t}$ denote the signal received by bidder $i$. Given $N_{t}=n$, let $S_{t}=\left(S_{1 t}, \ldots, S_{n t}\right)$ and $V_{t}=\left(V_{1 t}, \ldots, V_{n t},\right)$, where without loss we re-label bidders as firms $i=1, \ldots n$. For any conditioning set $\Omega \subseteq\left(X_{t}, Z_{t}, E_{t}\right)$, let $F_{S V}\left(S_{t}, V_{t} \mid N_{t}, \Omega\right)$ denote the conditional distribution of bidders' signals and valuations. We assume $F_{S V}\left(S_{t}, V_{t} \mid N_{t}, X_{t}, Z_{t}, E_{t}\right)$ satisfies standard smoothness, symmetry, affiliation, and nondegeneracy conditions (see Assumption 2 in the text). We assume that $Z_{t}$ alters the joint distribution of signals and valuations only through its effect

\footnotetext{
${ }^{57}$ In practice we represent the set of bidders for neighboring tracts more parsimoniously with the number of such bidders.

${ }^{58}$ In our application we consider an extension allowing a random reserve price.

${ }^{59} \mathrm{As}$ is standard in the literature, we assume that only bidders incurring the entry cost can submit a bid (see, e.g., Levin and Smith (1994), Li and Zheng (2009), Athey, Levin, and Seira (2011), Krasnokutskaya and Seim (2011), Gentry and Li (2014), or Bhattacharya, Roberts, and Sweeting (2014)). This assumption can be relaxed, allowing bidding with no signal, under an equilibrium selection rule specifying that firms indifferent to entry do not enter.
} 
on $N_{t}$, i.e.,

$$
F_{S V}\left(S_{t}, V_{t} \mid N_{t}, Z_{t}, X_{t}, E_{t}\right)=F_{S V}\left(S_{t}, V_{t} \mid N_{t}, X_{t}, E_{t}\right)
$$

and that $Z_{t}$ is independent of $E_{t}$ conditional on $X_{t}$. We discuss the justification for this conditional independence assumption below. We assume the multiplicatively separable structure

$$
V_{i t}=V_{i t}^{0} \lambda\left(X_{t}, E_{t}\right) \text {, }
$$

where the function $\lambda$ is bounded and the random variables $\left(V_{1 t}^{0}, \ldots, V_{n_{t} t}^{0}, S_{1 t}, \ldots, S_{n_{t} t}\right)$ are independent of $\left(X_{t}, E_{t}, Z_{t}\right)$ conditional on $N_{t}$. We assume that $\lambda\left(x, E_{t}\right)$ is continuously distributed for all $x \in \mathbb{X} .60$

Note that we have not restricted the dimension of $E_{t}$, imposed any monotonicity condition on $\lambda$, or required independence between $X_{t}$ and $E_{t}{ }^{61}$ Nonetheless, we can obtain the model of unobserved heterogeneity in the text by representing the random variable $\lambda\left(X_{t}, E_{t}\right)$ in terms of its quantiles conditional on $X_{t}$. In particular, given $X_{t}=x$, let $F_{\lambda}(\cdot \mid x)$ denote the $\mathrm{CDF}$ of the random variable $\lambda\left(x, E_{t}\right)$, and let

$$
U_{t}=F_{\lambda}\left(\lambda\left(x, E_{t}\right) \mid x\right)
$$

For $u \in[0,1]$ define $F_{\lambda}^{-1}(u \mid x)=\inf \left\{\lambda: F_{\lambda}(\lambda \mid x) \geq u\right\}$ and let

$$
\Gamma(x, u)=F_{\lambda}^{-1}(u \mid x)
$$

Combining A.2 and (A.3), for each $x$ we have $F_{\lambda}\left(\Gamma\left(x, U_{t}\right) \mid x\right)=U_{t}=F_{\lambda}\left(\lambda\left(x, E_{t}\right) \mid x\right)$,

\footnotetext{
${ }^{60}$ This assumption can be dropped, for example, by assuming instead that any indifferences in the entry stage are broken the same way conditional on observables.

${ }^{61}$ This may be important, as the nomination process by which tracts were offered for lease in our sample period suggests that a tract with "undesirable" value of $X_{t}$ may have been unlikely to be offered unless the value of $E_{t}$ made the tract desirable. See, e.g., Hendricks, Porter, and Boudreau (1987) for a discussion of the nomination process.
} 
i.e.,

$$
\Gamma\left(x, U_{t}\right)=\lambda\left(x, E_{t}\right) .
$$

By construction, $\Gamma$ is weakly increasing in its second argument, and $U_{t}$ is uniform on $[0,1]$ conditional on $X_{t}$. And because $U_{t}$ is a measurable function of $E_{t}$ conditional on $X_{t}, U_{t}$ is independent of $Z_{t}$ conditional on $X_{t}$.

Note that in this new representation of the model, the distribution of $U_{t}$ does not vary with $X_{t}$, but its interpretation generally will. Observe that because $\Gamma\left(x_{t}, u_{t}\right)=$ $\lambda\left(x_{t}, e_{t}\right)$ for all $t$ by construction, $\Gamma\left(X_{t}, U_{t}\right)$ fully characterizes the variation and dependence in valuations and bids that arises from the observables and unobservables. Likewise, controlling for the value of $\Gamma\left(X_{t}, U_{t}\right)$ fully controls for the effects of auction observables and unobservables $\left(X_{t}, E_{t}\right)$ on valuations, bids, and equilibrium first-order conditions. However, we will not be able to quantify the effect of a change in $X_{t}$ (or one of its components) holding unobservables fixed, since our $U_{t}$ is redefined at every value of $X_{t}$.

\section{A.2 Equilibrium}

We henceforth use the representation of the model just derived. The set of firms $\mathcal{I}$, the rules of the game, the values of $\left(X_{t}, U_{t}, Z_{t}\right)$, and the distribution $F_{S V}\left(S_{t}, V_{t} \mid N_{t}, X_{t}, U_{t}\right)$ are common knowledge among firms. We consider perfect Bayesian equilibrium in pure strategies, with weakly increasing strategies in the auction stage.

The second stage of the game is identical to the first-price sealed bid auction with symmetric affiliated values studied by Milgrom and Weber (1982), who characterize the unique Bayes-Nash equilibrium in increasing bidding strategies. Bidder $i$ 's payoff in the auction stage can be written as a function of the commonly known $\left(N_{t}, X_{t}, U_{t}\right)$

and the realized bidder signals $S_{t}$. As noted in the text, multiplicative separability of valuations is inherited by equilibrium bids. This implies that a bidder's ex post 
profit, denoted by $\pi\left(S_{i t}, S_{-i t}, N_{t}, X_{t}, U_{t}\right)$, is strictly increasing in the index $\Gamma\left(X_{t}, U_{t}\right)$ and, therefore, weakly increasing in $U_{t}$. Further, we assume the usual case in which the ex ante expected equilibrium payoff

$$
\bar{\pi}\left(N_{t}, X_{t}, U_{t}\right)=E\left[\pi\left(S_{i t}, S_{-i t}, N_{t}, X_{t}, U_{t}\right) \mid N_{t}, X_{t}, U_{t}\right]
$$

is strictly decreasing in $N_{t}{ }^{62}$

In the entry stage, firms make decisions based on the cost of entry and expected profit from participating in the auction. Let $C_{i t}=c_{i}\left(X_{t}\right)$. For firm $i$, entering when $n-1$ other firms will also enter implies expected profit

$$
\bar{\pi}\left(n, X_{t}, U_{t}\right)-C_{i t}
$$

Conditional on $\left(X_{t}, Z_{t}, U_{t}\right)$, and given equilibrium beliefs about payoffs in the auction stage, the entry stage is then equivalent to the entry game in Berry (1992). Berry showed that a pure strategy equilibrium exists and that with probability one all equilibria exhibit the same number of entrants, given by

$$
n^{E}\left(X_{t}, Z_{t}, U_{t}\right)=\max _{0 \leq n \leq I}\left\{n: \bar{\pi}\left(n, X_{t}, U_{t}\right)-C_{i t} \geq 0\right\}
$$

Recall that $\left(X_{t}, Z_{t}\right)$ determine the values of $\left\{C_{i t}\right\}_{i \in \mathcal{I}}$.

Thus, in any pure strategy perfect Bayesian equilibrium (with weakly increasing bidding) we have

$$
N_{t}=n^{E}\left(X_{t}, Z_{t}, U_{t}\right)
$$

Further, because $\bar{\pi}\left(N_{t}, X_{t}, U_{t}\right)$ is weakly increasing in $U_{t}$, so is the function $n^{E}$.

\footnotetext{
${ }^{62}$ We know of no counterexample to strict monotonicity in $N_{t}$ under the assumption that $U_{t}$ is the only latent source of dependence between the entry and auction stages - i.e., that Assumption 12 holds. Nonmonotonicity (within the relevant range of $N_{t}$ ) could lead to existence of multiple equilibria with different numbers of bidders.
} 


\section{A.3 The Instrument}

Our instrument for bidder entry $Z_{t}$ is the number of neighbor firms. First consider the "exclusion" requirement (Assumption 5). We have assumed directly that $Z_{t}$ is independent of $\left(S_{t}, V_{t}^{0}\right)$ conditional on $N_{t}$, i.e., that $X_{t}$ are the only observables directly affecting bidder valuations ${ }^{63}$ However we must verify that $Z_{t}$ is also independent of $U_{t}$ conditional on $X_{t}$. A tract with three neighbor leases, for example, may have one, two, or three neighbor firms, depending on which bidders for the neighboring leases won those auctions. Given the number of neighbor leases and the bidders for each neighbor tract (i.e., conditional on $X_{t}$ ), the number of distinct winners reflects only random variation in bidders' signals at prior auctions. Recall that signals are assumed independent of tract-specific unobserved heterogeneity and independent across tracts. Thus, even in the case of spatially correlated tract-level unobservables $E_{t}$, the conditional independence requirement $Z_{t} \Perp U_{t} \mid X_{t}$ will hold.

Regarding the "relevance" requirement for the instrument $Z_{t} 6$ observe that changes in the number of neighbor firms affects entry because for some combinations of $\left(X_{t}, Z_{t}, U_{t}\right)$ the market will accommodate the $n+1$ st entrant only if there is a potential bidder with low signal acquisition cost. For example, we will sometimes have two entrants because the market would support entry by a third (low cost) neighbor, but not by a third firm that is a (high cost) non-neighbor. Thus, larger values of $Z_{t}$ will lead, all else equal, to weakly larger numbers of entrants. If the cost asymmetry is substantial, the effects of the instrument on entry will be substantial as well.

\footnotetext{
${ }^{63}$ This could fail here if the number of neighbor firms had a direct effect on tract value (given $X_{t}$ ), e.g., by driving up costs of negotiating production from common pools.

${ }^{64}$ This discussion is informal. We have specific "relevance" requirements in the identification results. Our instrument falls short of these by being discrete rather than continuous. As discussed in the identification analysis, discrete instruments may lead to reliance on functional form to interpolate between the points at which the index function $\gamma(x, \cdot)$ is identified at each $x$.
} 


\section{A.4 Truncation}

In the OCS data we observe no information about (even existence of) leases offered for sale but attracting no bids. Given the tract nomination process in place during the sample period, this may not have been a frequent phenomenon. But existence of such leases could imply a form of selection on unobservables: leases attracting no bids would be those with relatively undesirable unobservables. Here we demonstrate that such selection is accommodated by interpreting the unobservable in our model as that conditioned on the event that the auction attracts at least one bid.

In our model, offered leases attracting no bids are those for which

$$
\pi\left(1, x_{t}, u_{t}\right) \leq c_{i t} \quad \forall i \in \mathcal{I}
$$

Letting $\pi^{-1}(c ; 1, x)=\sup \{u: \pi(1, x, u)<c\}$, we can rewrite A.4 as

$$
u_{t} \leq \pi^{-1}\left(\min _{i \in \mathcal{I}} c_{i}\left(x_{t}\right) ; 1, x_{t}\right)
$$

or, more simply given our definition of $c_{i}\left(x_{t}\right)$,

$$
u_{t} \leq \underline{u}\left(x_{t}\right)
$$

Recalling that the definition of $U_{t}$ already changes with each value of $X_{t}$, we obtain the original model by redefining $U_{t}$ to denote the value of the unobservable conditional on truncation at $\underline{u}\left(X_{t}\right) 66$

\footnotetext{
${ }^{65}$ Note that the threshold for attracting a single bidder varies depending on the presence of at least one neighbor tract (firm), but not with the number of neighbor firms $Z_{t}$.

${ }^{66}$ More formally, going back to the original formulation of the unobserved heterogeneity in terms of $E_{t}$, let $\tilde{\pi}\left(N_{t}, X_{t}, E_{t}\right)$ denote the expected second-stage payoff for a bidder facing $N_{t}-1$ opponents given $\left(X_{t}, E_{t}\right)$. Under the separable structure A.1), we have $\tilde{\pi}\left(N_{t}, X_{t} \cdot E_{t}\right)=\tilde{\pi}^{0}\left(N_{t}\right) \lambda\left(X_{t}, E_{t}\right)$. Given $X_{t}=x$, we have a zero-bidder auction when $\lambda\left(x, E_{t}\right) \leq \frac{\min _{i} c_{i}\left(x_{t}\right)}{\tilde{\pi}^{0}(1)}$. Thus, we modify the construction of $U_{t}$ by letting $F_{\lambda}(\cdot \mid x)$ denote the CDF of $\lambda\left(x, E_{t}\right)$ conditional on $\lambda\left(x, E_{t}\right)>\frac{\min _{i} c_{i}\left(x_{t}\right)}{\tilde{\pi}^{0}(1)}$.
} 


\section{B Proofs Omitted from the Text}

Here we provide proofs omitted from the text. For convenience we restate the results being proved.

Corollary 1. Under Assumptions 1 6, the distribution of $U_{t} \mid\left(X_{t}, N_{t}\right)$ is identified.

Proof.

We can express $\operatorname{Pr}\left(U_{t} \leq u \mid X_{t}=x, N_{t}=n\right)$ as

$$
F_{U \mid X N}(u \mid x, n)=\int F_{U \mid X Z N}(u \mid x, z, n) d \zeta(z \mid x, n)
$$

where $F_{U \mid X Z N}$ is the distribution of $U_{t} \mid\left(X_{t}, Z_{t}, N_{t}\right)$ and $\zeta$ is the distribution of $Z_{t} \mid\left(X_{t}, N_{t}\right)$. Conditional on $N_{t}=n, Z_{t}=z$, and $X_{t}=x, U_{t}$ is uniform on $\left[\tau_{n-1}(x, z), \tau_{n}(x, z)\right]$, and by Theorem 1 the endpoints $\tau_{n-1}(x, z)$ and $\tau_{n}(x, z)$ are identified. So $F_{U \mid X Z N}$ is known. Since $\zeta$ is directly observed, the result follows from (B.1).

Lemma 2. Under Assumptions 1 8, for all $n \geq \underline{n}$, all $(x, z) \in \mathbb{Y}(n)$, and all $\left(x^{\prime}, z^{\prime}\right) \in$ $\mathbb{Y}(n), \gamma\left(x, \tau_{n}(x, z)\right)-\gamma\left(x^{\prime}, \tau_{n-1}\left(x^{\prime}, z^{\prime}\right)\right)$ is identified.

Proof. For $n^{*}$ as defined in Assumption 8 , take $n \leq n^{*}$ and let $x(n), z(n)$, and $\hat{z}(n)$ be as in part (i) of Assumption 8 so that

$$
\begin{aligned}
& \underline{n}(x(n), z(n))=n \\
& \underline{n}(x(n), \hat{z}(n))=n+1 .
\end{aligned}
$$

Since $(x(n), z(n)) \in \mathbb{Y}(n)$ and $(x(n), \hat{z}(n)) \in \mathbb{Y}(n+1)$, Lemma 1 implies identification of

$$
\gamma\left(x^{\prime}, \tau_{n-1}\left(x^{\prime}, z^{\prime}\right)\right)-\gamma\left(x(n), \tau_{n-1}(x(n), z(n))\right)
$$

and

$$
\gamma\left(x^{\prime \prime}, \tau_{n}\left(x^{\prime \prime}, z^{\prime \prime}\right)\right)-\gamma\left(x(n), \tau_{n}(x(n), \hat{z}(n))\right)
$$


for all $\left(x^{\prime}, z^{\prime}\right) \in \mathbb{Y}(n)$ and $\left(x^{\prime \prime}, z^{\prime \prime}\right) \in \mathbb{Y}(n+1)$. By (4) and (B.2),

$$
\tau_{n-1}(x(n), z(n))=0=\tau_{n}(x(n), \hat{z}(n)),
$$

so subtracting $(\mathrm{B} .3)$ from $(\mathrm{B} .4)$ yields identification of

$$
\gamma\left(x^{\prime \prime}, \tau_{n}\left(x^{\prime \prime}, z^{\prime \prime}\right)\right)-\gamma\left(x^{\prime}, \tau_{n-1}\left(x^{\prime}, z^{\prime}\right)\right)
$$

for all $\left(x^{\prime \prime}, z^{\prime \prime}\right) \in \mathbb{Y}(n+1)$ and $\left(x^{\prime}, z^{\prime}\right) \in \mathbb{Y}(n)$. By Assumption 7, there exists some $\left(x^{\prime \prime}, z^{\prime \prime}\right)$ that is in both $\mathbb{Y}(n+1)$ and $\mathbb{Y}(n)$. The claim then follows from Lemma 1 . A symmetric argument applies for $n>n^{*} 67$

Lemma 3. Let Assumptions 18 hold. Then for all $n \geq \underline{n}$ and all $(x, z) \in \mathbb{Y}(n)$, the values of $\gamma\left(x, \tau_{n-1}(x, z)\right)$ and $\gamma\left(x, \tau_{n}(x, z)\right)$ are identified.

Proof. We proceed by induction, starting with $n=\underline{n}$. By the normalization (1), $\gamma\left(x^{0}, 0\right)=0$, where for some $z$ we have $\left(x^{0}, z\right) \in \mathbb{Y}(\underline{n})$. Lemma 1 then implies identification of $\gamma\left(x, \tau_{\underline{n}-1}(x, z)\right)$ for all $(x, z) \in \mathbb{Y}(\underline{n})$. Lemma 2 then implies identification of $\gamma\left(x, \tau_{\underline{n}}(x, z)\right)$ for all $(x, z) \in \mathbb{Y}(\underline{n})$. Now take any $n>\underline{n}$ and suppose that $\gamma\left(x, \tau_{n-1}(x, z)\right)$ is known for all $(x, z) \in \mathbb{Y}(n-1)$. By Assumption 7 there exists a point $(\tilde{x}, \tilde{z})$ in $\mathbb{Y}(n-1) \cap \mathbb{Y}(n)$. Since we have already identified $\gamma\left(\tilde{x}, \tau_{n-1}(\tilde{x}, \tilde{z})\right)$, by Lemma 1 we also know the value of $\gamma\left(x, \tau_{n-1}(x, z)\right)$ for all $(x, z)$ in $\mathbb{Y}(n)$. By Lemma 2. this implies identification of $\gamma\left(x, \tau_{n}(x, z)\right)$ for all $(x, z)$ in $\mathbb{Y}(n)$.

Lemma 4. Under Assumptions $11, \tau_{n-1}\left(X_{t}, Z_{t}\right)$ is continuous in $Z_{t}$ on the pre-image

\footnotetext{
${ }^{67}$ Note that the arguments used to show Lemmas 1 and 2 will often imply several forms of overidentification. For example, Lemma 1 implies overidentification of $\gamma\left(x^{\prime}, \tau_{m}\left(x^{\prime}, z^{\prime}\right)\right)-\gamma\left(x, \tau_{m}(x, z)\right)$ for any $m$ which is both smaller than $\min \left\{\bar{n}\left(x^{\prime}, z^{\prime}\right), \bar{n}(x, z)\right\}$ and larger than $\max \left\{\underline{n}\left(x^{\prime}, z^{\prime}\right), \underline{n}(x, z)\right\}$. And while Assumption 7 ensures only that there exist one $(\tilde{x}, \tilde{z}) \in \mathbb{Y}(n)$ that is also in $\mathbb{Y}(n-1)$, when there is more than one such pair the proof of Lemma 2 will provide multiple ways of constructing the same value of a given difference $\gamma\left(x^{\prime \prime}, \tau_{n}\left(x^{\prime \prime}, z^{\prime \prime}\right)\right)-\gamma\left(x^{\prime}, \tau_{n-1}\left(x^{\prime}, z^{\prime}\right)\right)$. Finally, in practice there may often be more than one value of $n^{*}$ satisfying Assumption 8, resulting in some duplication in the differences identified in the two halves of the proof of Lemma 2 .
} 
of $(0,1)$.

Proof. Fix $n, x$, and $z$ such that $\tau_{n-1}(x, z) \in(0,1)$. Let $\tau=\tau_{n-1}(x, z)$ and let $\nu>0$ be sufficiently small that $\tau+\nu<1$ and $\tau-\nu>0$. We show that for any such $\nu$ there exists $\epsilon>0$ such that for every $z^{\prime}$ satisfying $\left\|z^{\prime}-z\right\|<\epsilon$ we have $\tau_{n-1}\left(x, z^{\prime}\right) \in(\tau-\nu, \tau+\nu)$. Let $\delta=\nu / 2$. By the definition of $\tau_{n-1}(x, z)$ and weak monotonicity of $\eta$ in $U_{t}, \eta(x, z, \tau-\delta)<n$. So by Assumption 9 there exists $\epsilon_{1}>0$ such that for any $z^{\prime}$ satisfying $\left\|z^{\prime}-z\right\|<\epsilon_{1}, \eta\left(x, z^{\prime}, \tau^{\prime}\right)<n$ for some $\tau^{\prime} \in(\tau-2 \delta, \tau)$. Similarly, because $\eta(x, z, \tau+\delta) \geq n$, Assumption 9 ensures that there exists $\epsilon_{2}>0$ such that for any $z^{\prime}$ satisfying $\left\|z^{\prime}-z\right\|<\epsilon_{2}, \eta\left(x, z^{\prime}, \tau^{\prime \prime}\right) \geq n$ for some $\tau^{\prime \prime} \in(\tau, \tau+2 \delta)$. Letting $\epsilon=\min \left\{\epsilon_{1}, \epsilon_{2}\right\}$, we have shown that for any $z^{\prime}$ satisfying $\left\|z^{\prime}-z\right\|<\epsilon$, $\eta\left(x, z^{\prime}, \tau^{\prime}\right)<n$ for some $\tau^{\prime} \in(\tau-\nu, \tau)$ while $\eta\left(x, z^{\prime}, \tau^{\prime \prime}\right) \geq n$ for some $\tau^{\prime \prime} \in(\tau, \tau+\nu)$. At such $z^{\prime}, \tau_{n-1}\left(x, z^{\prime}\right)$ must lie in $\left[\tau^{\prime}, \tau^{\prime \prime}\right]$.

\section{Random Reserve Price}

As discussed in section 7.1, the MMS occasionally exercised its right to reject all bids, typically when the number or level of bids received was low. Following Hendricks, Porter, and Spady (1989) and Hendricks, Porter, and Wilson (1994), we model this rejection policy by assuming that at each auction $t$ the MMS used a random reserve price $R_{t}$ whose realization was unknown to bidders.

We let the distribution of $R_{t}$ vary with the number of bidders and with the auction characteristics $\left(X_{t}, U_{t}\right)$. The dependence on $\left(X_{t}, U_{t}\right)$ is assumed to mirror that of tract valuations. Thus, we assume

$$
R_{t}=R_{t}^{0} \times \Gamma\left(X_{t}, U_{t}\right)
$$

where $R_{t}^{0}$ is independent of $\left(X_{t}, U_{t}, S_{t}, V_{t}\right)$ conditional on $N_{t}$. In this formulation, a 
homogenized winning bid $M_{t}^{0}$ is accepted if and only if $M_{t}^{0} \geq R_{t}^{0}$. Let $H_{R}\left(\cdot \mid N_{t}\right)$ denote the distribution of $R_{t}^{0}$ given $N_{t}$.

Following Bajari and Hortaçsu (2003), for each value of $n$ we model the distribution $H_{R}(\cdot \mid n)$ using the marginal distribution of homogenized bids in an $n$-bidder auction. However, to fit the data, we allow the MMS "bid" to be less aggressive than a real bid by introducing a scaling factor $\sigma_{n} 68$ In particular, we assume

$$
H_{R}(r \mid n)=\min \left\{1, G_{B^{0}}\left(\frac{r}{\sigma_{n}} ; n\right)\right\}
$$

Thus, the random reserve price has the same distribution as a rescaled bid.

Motivated by the observed rejection frequencies (see Table 13 below), we estimate separate scaling factors for $n=1, n \in\{2,3\}$ and $n \geq 4$. Taking the estimates of $\theta=\left(\theta_{\tau}, \theta_{\gamma}, \theta_{b}\right)$ as given, we fit these scaling parameters $\sigma$ to the observed bid acceptance decisions

$$
Y_{i t}=1\left\{M_{i t} \geq R_{t}\right\}
$$

using the quasi-likelihood function

$$
\begin{aligned}
& \mathcal{L}(y ; \sigma, \hat{\theta})= \\
& \prod_{t=1}^{T} \frac{1}{\tau_{n_{t}}\left(x_{t}, z_{t} ; \hat{\theta}_{\tau}\right)-\tau_{n_{t}-1}\left(x_{t}, z_{t} ; \hat{\theta}_{\tau}\right)} \int_{\tau_{n_{t}-1}\left(x_{t}, z_{t} ; \hat{\theta}_{\tau}\right)}^{\tau_{n_{t}}\left(x_{t}, z_{t} ; \hat{\theta}_{\tau}\right)} H_{R}\left(m_{t}-\gamma\left(x_{t}, u ; \hat{\theta}_{\gamma}\right) \mid n_{t} ; \hat{\theta}_{b}, \sigma\right)^{y_{t}} \times \\
& \left.\left(1-H_{R}\left(m_{t}-\gamma\left(x_{t}, u ; \hat{\theta}_{\gamma}\right) \mid n_{t} ; \hat{\theta}_{b}, \sigma\right)\right)^{1-y_{t}} d u . \quad \text { (C. } 1\right)
\end{aligned}
$$

Table 13 shows the actual and fitted bid acceptance rates for each value of $n$.

Introducing the random reserve price requires only a minor change in the inversion of bidder first-order conditions. Under the null hypothesis of private values, bidders'

\footnotetext{
${ }^{68}$ Very similar results are obtained fitting a lognormal distribution for $R_{t}^{0}$ or allowing the homogenized reserve price to be correlated with the homogenized bids.
} 
Table 13: Random Reserve Model Fit

\begin{tabular}{c|cc}
\hline & \multicolumn{2}{|c}{ acceptance rate } \\
$n$ & actual & fitted \\
\hline 1 & 0.921 & 0.917 \\
2 & 0.978 & 0.981 \\
3 & 0.997 & 0.991 \\
4 & 0.991 & 0.997 \\
5 & 1.000 & 0.995 \\
6 & 1.000 & 0.998 \\
7 & 1.000 & 0.998 \\
8 & 1.000 & 0.998 \\
9 & 1.000 & 0.997 \\
10 & 1.000 & 0.999 \\
$11-18$ & 1.000 & 0.999 \\
\hline
\end{tabular}

homogenized pivotal expected values are identified using a revised first-order condition

$$
w^{0}\left(s_{i t} ; n_{t}\right)=b_{i t}^{0}+\frac{G_{M^{0} \mid B^{0}}\left(b_{i t}^{0} ; b_{i t}^{0}, n_{t}\right) H_{R}\left(b_{i t}^{0} ; n_{t}\right)}{G_{M^{0} \mid B^{0}}\left(b_{i t}^{0} ; b_{i t}^{0}, n_{t}\right) h_{R}\left(b_{i t}^{0} ; n_{t}\right)+g_{M^{0} \mid B^{0}}\left(b_{i t}^{0} ; b_{i t}^{0}, n_{t}\right) H_{R}\left(b_{i t}^{0} ; n_{t}\right)} .
$$

This allows testing of the private values null as in the baseline model.

An nuance here is that under the alternative of common values, the right-hand side of C.2 is not equal to $w^{0}\left(s_{i t}, s_{i t} ; n_{t}\right)$ but to a weighted averag€ 69

$$
\varphi\left(s_{i t}, n_{t}\right) \underline{w}^{0}\left(s_{i t} ; n_{t}\right)+\left[1-\varphi\left(s_{i t}, n_{t}\right)\right] w^{0}\left(s_{i t} ; n_{t}\right),
$$

where

$$
\underline{w}^{0}\left(s_{i t} ; n_{t}\right)=E\left[V_{i t}^{0} \mid S_{i t}=s_{i t}, \max _{j \neq i} S_{j t} \leq s_{i t}, N_{t}=n_{t}\right]
$$

denotes a bidder's expected (homogenized) valuation conditional on his signal and

${ }^{69}$ The weight $\varphi\left(s_{i t}, n_{t}\right)$ is equal to

$$
h\left(b_{i t}^{0}, n_{t}\right) G_{M \mid B}\left(b_{i t}^{0} ; b_{i t}^{0}, n_{t}\right) /\left[h\left(b_{i t}^{0}, n_{t}\right) G_{M \mid B}\left(b_{i t}^{0} ; b_{i t}^{0}, n_{t}\right)+H\left(b_{i t}^{0}, n_{t}\right) g_{M \mid B}\left(b_{i t}^{0} ; b_{i t}^{0}, n_{t}\right)\right] .
$$

Observe that all weight is placed on the pivotal expected value when the rejection probability $1-H\left(b_{i t}^{0}, n_{t}\right)$ is zero. 
on winning the auction. This expectation is slightly different from the homogenized pivotal expected value. And although both $\underline{w}^{0}\left(s_{i t} ; n_{t}\right)$ and $w^{0}\left(s_{i t} ; n_{t}\right)$ are decreasing in $n_{t}$ in a common values model, the weights $\varphi\left(s_{i t}, n_{t}\right)$ also vary with $n_{t}$, leaving an ambiguous prediction regarding how the distribution of the weighted average varies with $n_{t}$. This implies that violations of the private values null might fail to reveal themselves, and further that there may be no relationship between the estimated distributions obtained from the first-order condition that can be explained only by violation of the model's maintained hypotheses.

Given the stochastic ordering we do find, this caveat implies that the evidence we obtain in favor of common values from this specification is conditioned on a maintained assumption that our model is correctly specified. Such conditioning is typical in hypothesis testing but unlike the baseline specification, where we could partition the set of all possible outcomes to those consistent with private values, those consistent with common values, and those inconsistent with the model. 


\section{Additional Results and Tables}

\section{D.1 Baseline Specification Fine Binning Test Results}

Table 14: Test p-values

Baseline Specification, Fine Binning

Test for Common Values

\begin{tabular}{l|c|c} 
& With UH & No UH \\
\hline 2 vs. 3 & 0.001 & 0.046 \\
3 vs. 4 & 0.212 & 0.703 \\
4 vs. 5 & 0.662 & 0.869 \\
5 vs. 6 & 0.625 & 0.733 \\
6 vs. 7 & 0.073 & 0.270 \\
Max & 0.019 & 0.541
\end{tabular}

$\underline{\text { Specification Test }}$

\begin{tabular}{l|c|c} 
& With UH & No UH \\
\hline 2 vs. 3 & 1.000 & 1.000 \\
3 vs. 4 & 0.730 & 0.229 \\
4 vs. 5 & 0.567 & 0.086 \\
5 vs. 6 & 0.627 & 0.249 \\
6 vs. 7 & 0.562 & 0.440 \\
Max & 0.922 & 0.239 \\
\hline
\end{tabular}

\section{D.2 Parameter Estimates: Alternative Specifications}


Table 15: Entry Model Estimates

Alternative Specifications

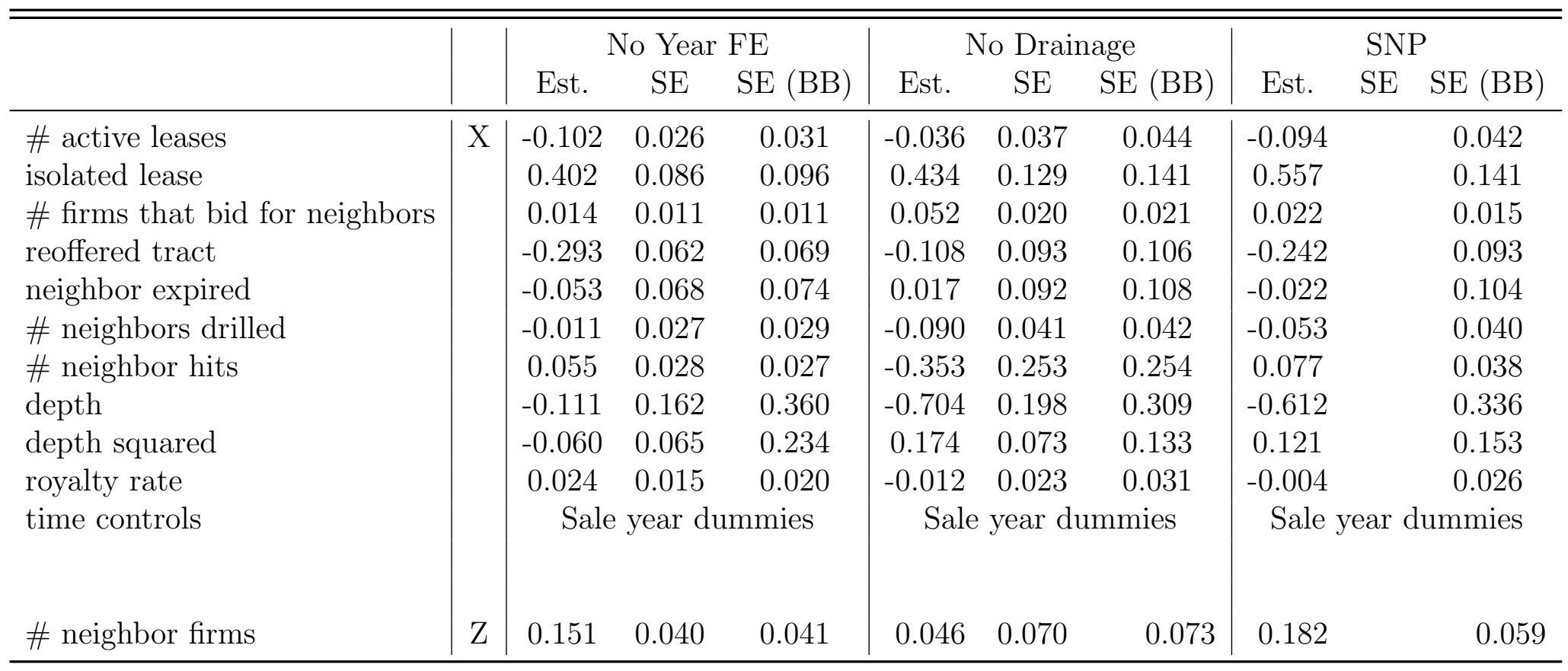


Table 16: Index Function Estimates Alternative Specifications

\begin{tabular}{|c|c|c|c|c|c|c|c|c|c|c|}
\hline & & \multicolumn{3}{|c|}{ SNP } & \multicolumn{3}{|c|}{ No time FE } & \multicolumn{3}{|c|}{ No Drainage } \\
\hline & & Est. & $\mathrm{SE}$ & $\mathrm{SE}(\mathrm{BB})$ & Est. & $\mathrm{SE}$ & $\mathrm{SE}(\mathrm{BB})$ & Est. & $\mathrm{SE}$ & $\mathrm{SE}(\mathrm{BB})$ \\
\hline$\#$ active leases & \multirow[t]{11}{*}{$\mathrm{X}$} & 0.013 & 0.018 & 0.022 & -0.006 & 0.027 & 0.027 & 0.006 & 0.023 & 0.030 \\
\hline isolated lease & & 0.060 & 0.060 & 0.091 & 0.027 & 0.079 & 0.111 & 0.048 & 0.085 & 0.123 \\
\hline \# firms that bid for neighbors & & 0.032 & 0.008 & 0.011 & 0.052 & 0.010 & 0.013 & 0.036 & 0.013 & 0.019 \\
\hline reoffered tract & & -0.182 & 0.048 & 0.070 & -0.245 & 0.078 & 0.091 & -0.102 & 0.064 & 0.093 \\
\hline neighbor expired & & -0.277 & 0.047 & 0.068 & -0.119 & 0.062 & 0.083 & -0.203 & 0.060 & 0.087 \\
\hline \# neighbors drilled & & 0.047 & 0.020 & 0.025 & 0.122 & 0.027 & 0.028 & 0.058 & 0.029 & 0.042 \\
\hline \# neighbor hits & & -0.021 & 0.020 & 0.026 & -0.047 & 0.028 & 0.031 & -0.096 & 0.177 & 0.219 \\
\hline depth & & -0.248 & 0.137 & 0.274 & 1.203 & 0.146 & 0.365 & -0.442 & 0.160 & 0.297 \\
\hline depth squared & & 0.094 & 0.065 & 0.120 & -0.307 & 0.064 & 0.186 & 0.161 & 0.071 & 0.124 \\
\hline royalty rate & & -0.007 & 0.010 & 0.017 & 0.029 & 0.015 & 0.015 & 0.015 & 0.013 & 0.023 \\
\hline time controls & & \multicolumn{3}{|c|}{ Sale year dummies } & \multicolumn{3}{|c|}{ Sale year dummies } & \multicolumn{3}{|c|}{ Sale year dummies } \\
\hline unobserved heterogeneity & $\mathrm{U}$ & 1.319 & 0.291 & 0.405 & 1.989 & 0.584 & 0.646 & 0.941 & 0.358 & 0.513 \\
\hline
\end{tabular}


Table 17: Copula Correlation Estimates Alternative Specifications

\begin{tabular}{r|ccc|ccc|ccc}
\hline \hline & \multicolumn{3}{|c|}{ SNP } & \multicolumn{3}{c|}{ No time FE } & \multicolumn{3}{c}{ No Drainage } \\
$n$ & Est. & SE & SE (BB) & Est. & SE & SE (BB) & Est. & SE & SE (BB) \\
\hline 2 & 0.043 & 0.041 & 0.050 & 0.197 & 0.049 & 0.051 & 0.024 & 0.056 & 0.060 \\
3 & 0.031 & 0.030 & 0.040 & 0.212 & 0.040 & 0.042 & 0.007 & 0.033 & 0.047 \\
4 & 0.090 & 0.031 & 0.032 & 0.275 & 0.038 & 0.038 & 0.053 & 0.034 & 0.036 \\
5 & 0.136 & 0.030 & 0.041 & 0.301 & 0.031 & 0.042 & 0.111 & 0.035 & 0.042 \\
6 & 0.112 & 0.035 & 0.037 & 0.259 & 0.032 & 0.042 & 0.083 & 0.034 & 0.036 \\
7 & 0.111 & 0.044 & 0.043 & 0.342 & 0.061 & 0.041 & 0.090 & 0.043 & 0.040 \\
8 & 0.133 & 0.039 & 0.040 & 0.261 & 0.039 & 0.052 & 0.160 & 0.052 & 0.046 \\
9 & 0.295 & 0.071 & 0.067 & 0.400 & 0.076 & 0.073 & 0.290 & 0.071 & 0.082 \\
10 & 0.158 & 0.051 & 0.068 & 0.307 & 0.055 & 0.055 & 0.155 & 0.053 & 0.063 \\
$11-18$ & 0.108 & 0.025 & 0.020 & 0.246 & 0.029 & 0.031 & 0.098 & 0.026 & 0.026 \\
\hline
\end{tabular}




\section{E Additional Computational Detail}

Here we discuss additional computational aspects of our estimation procedure. A useful feature of the Bernstein polynomial specification is the ease of imposing otherwise complex functional restrictions through linear restrictions on the vector of Bernstein coefficients. For instance, a necessary and sufficient condition for the function to integrate to one is that $\sum_{j=0}^{m} \theta_{b, n}^{(j)}=m+1$. Additionally, the Bernstein polynomials allow easy transformation between the density and associated cumulative distribution. In particular,

$$
\tilde{G}_{B_{i}^{0}}\left(\tilde{b}^{0} ; \tilde{\theta}_{b}, n\right)=\sum_{j=0}^{m+1} \tilde{\theta}_{b, n}^{(j)} q_{j, m+1}\left(\Phi\left(\tilde{b}^{0}\right)\right)
$$

where $\tilde{\theta}_{b, n}=\left(\tilde{\theta}_{b, n}^{(0)}, \ldots, \tilde{\theta}_{b, n}^{(m+1)}\right)^{\prime}=M \theta_{b, n}$ for a known matrix $M$, and $\tilde{\theta}_{b}=\left\{\tilde{\theta}_{b, n}\right\}_{n=\underline{n}}^{\bar{n}}$. This is useful because we require the CDF of the bid marginal when applying the copula $\chi\left(\cdot ; \rho_{n}\right)$ to compute the joint density in $(13)$. In contrast to numerical integration, the transformation from density to CDF with Bernstein polynomials involves only a linear transformation of parameters and is exact.

One key computational issue is how one can derive tractable expressions for the conditional distributions that appear in the bid first-order condition (15). Below we show how the Gaussian copula can be leveraged to write $\tilde{G}_{M \mid B}\left(\tilde{b}_{i t}^{0} \mid \tilde{b}_{i t}^{0}, n\right)$ and $\tilde{g}_{M \mid B}\left(\tilde{b}_{i t}^{0} \tilde{b}_{i t}^{0}, n\right)$ using integrals of known normal distributions, which are easily computed using readily available methods. We first derive an expression for $\tilde{G}_{M \mid B}\left(\tilde{b}_{i t}^{0} \mid \tilde{b}_{i t}^{0}, n\right)$. An expression for $\tilde{g}_{M \mid B}\left(\tilde{b}_{i t}^{0} \mid \tilde{b}_{i t}^{0}, n\right)$ follows naturally.

First, note that

$$
\begin{aligned}
\tilde{G}_{M \mid B}(b \mid b, n) & =\operatorname{Pr}\left(\tilde{B}_{2 t}^{0} \leq b, \ldots, \tilde{B}_{n t}^{0} \leq b \mid \tilde{B}_{1 t}^{0}=b\right) \\
& =\operatorname{Pr}\left(\Phi^{-1}\left(\tilde{G}_{B_{i}^{0}}\left(\tilde{B}_{2 t}^{0}\right)\right) \leq b^{\star}, \ldots, \Phi^{-1}\left(\tilde{G}_{B_{i}^{0}}\left(\tilde{B}_{n t}^{0}\right)\right) \leq b^{\star} \mid \Phi^{-1}\left(\tilde{G}_{B_{i}^{0}}\left(\tilde{B}_{1 t}^{0}\right)\right)=b^{\star}\right)
\end{aligned}
$$


where $b^{\star}=\Phi^{-1}\left(\tilde{G}_{B_{i}^{0}}(b)\right)$ and we drop the dependence of $\tilde{G}_{B_{i}^{0}}$ on $\tilde{\theta}_{b}$ and $n$ for notational convenience. The second equality holds for every $b \in \mathbb{R}$, since the estimated $\tilde{G}_{B_{i}^{0}}$ has always full support.

Second, note that the Gaussian copula parametrization of the joint distribution of homogenized bids implies

$$
\left(\Phi^{-1}\left(\tilde{G}_{B_{i}^{0}}\left(\tilde{B}_{1 t}^{0}\right)\right), \ldots, \Phi^{-1}\left(\tilde{G}_{B_{i}^{0}}\left(\tilde{B}_{n t}^{0}\right)\right)\right) \sim N\left(0, \Sigma_{\rho_{n}}\right)
$$

where $\Sigma_{\rho_{n}}$ is the covariance matrix with constant pairwise correlation $\rho_{n}$.

Finally, the conditional normal distribution is also normal, so we can re-write the conditional probability E.2) as

$$
\tilde{G}_{M \mid B}(b \mid b, n)=\tilde{\Phi}\left(\Phi^{-1}\left(\tilde{G}_{B_{i}^{0}}(b)\right), \ldots, \Phi^{-1}\left(\tilde{G}_{B_{i}^{0}}(b)\right)\right)
$$

where $\tilde{\Phi}(\cdot)$ is the multivariate Normal CDF with mean $1_{(n-1) \times 1} \rho_{n} \Phi^{-1}\left(\tilde{G}_{B_{i}^{0}}(b)\right)$ and variance $\Sigma_{\rho_{n}}-\rho_{n}{ }^{2}$. Therefore computation of $\tilde{G}_{M \mid B}(b \mid b, n)$ requires only the evaluation of a known normal CDF.

This procedure naturally suggests one way of computing $\tilde{g}_{M \mid B}(b \mid b, n)$ as a multivariate integral. By definition,

$$
\tilde{g}_{M \mid B}(b \mid b, n)=\frac{d \tilde{\Phi}\left(\Phi^{-1}\left(\tilde{G}_{B_{i}^{0}}(b)\right), \ldots, \Phi^{-1}\left(\tilde{G}_{B_{i}^{0}}(b)\right)\right)}{d b}
$$

By symmetry and using the Leibniz integral rule,

$$
\begin{aligned}
& \tilde{g}_{M \mid B}(b \mid b, n)= \\
& (n-1) \frac{\tilde{g}_{B_{i}^{0}}(b)}{\phi\left(\tilde{G}_{B_{i}^{0}}(b)\right)} \int_{-\infty}^{\Phi^{-1}\left(\tilde{G}_{B_{i}^{0}}(b)\right)} \int_{-\infty}^{\Phi^{-1}\left(\tilde{G}_{B_{i}^{0}}(b)\right)} \tilde{\phi}\left(\Phi^{-1}\left(\tilde{G}_{B_{i}^{0}}(b)\right), b_{3}, \ldots, b_{n}\right) d b_{3} \ldots b_{n} .
\end{aligned}
$$


where we drop the dependence of $\tilde{g}_{B_{i}^{0}}$ on $\theta_{b}$ and $n$. The integral above is over a known multivariate normal density. It can be computed quickly and reliably using the algorithm suggested by Genz (1992). 


\section{References}

Akerlof, G. A. (1970): "The Market for Lemons: Quality Uncertainty and the Market Mechanism," The Quarterly Journal of Economics, 84, 488-500.

Aradillas-Lopez, A., P. A. Haile, K. Hendricks, and R. H. Porter (2017): "Testing Competition in U.S. Offshore Oil and Gas Lease Auctions," Working Paper, Penn State University.

Armstrong, T. B. (2013): "Bounds in Auctions with Unobserved Heterogeneity," Quantitative Economics, 4, 377-415.

Athey, S., And P. A. Haile (2002): "Identification of Standard Auction Models," Econometrica, 70, 2107-2140.

(2006): "Empirical Models of Auctions," in Advances in Economics and Econometrics, Theory and Applications: Ninth World Congress of the Econometric Society, ed. by R. Blundell, W. Newey, and T. Persson, vol. 2. Cambridge University Press.

(2007): "Nonparametric Approaches to Auctions," in Handbook of Econometrics, ed. by J. J. Heckman, and E. Leamer, vol. 6A, pp. 3847-3965. Elsevier.

Athey, S., J. Levin, And E. Seira (2011): "Comparing Open and Sealed Bid Auctions: Evidence from Timber Auctions," Quarterly Journal of Economics, 126, $207-257$.

Bajari, P., And A. Hortaçsu (2003): "The Winner's Curse, Reserve Prices, and Endogenous Entry: Empirical Insights from eBay Auctions," RAND Journal of Economics, 34, 329-355.

Balat, J. (2011): "Highway Procurement and the Stimulus Package: Identification and Estimation of Dynamic Auctions with Unobserved Heterogeneity," working paper, Yale University.

Berry, S. T. (1992): "Estimation of a Model of Entry in the Airline Industry," Econometrica, 60(4), 889-917.

Bhattacharya, V., J. Roberts, and A. Sweeting (2014): "Regulating Bidder Participation in Auctions," RAND Journal of Economics, 45, 675-704.

Bresnahan, T., and P. Reiss (1991): "Entry and Competition in Concentrated Markets," Journal of Political Economy, 99, 977-1009.

Campo, S., E. Guerre, I. M. Perrigne, and Q. Vuong (2011): "Semiparametric Estimation of First-Price Auctions with Risk Averse Bidders," Review of Economic Studies, 78, 112-147. 
Campo, S., I. M. Perrigne, and Q. Vuong (2003): "Asymmetry in First-Price Auctions with Affiliated Private Values," Journal of Applied Econometrics, 18, 197-207.

Chen, X., Y. Fan, and V. Tsyrennikov (2006): "Efficient Estimation of Semiparametric Multivariate Copula Models," Journal of the American Statistical Association, 101, 1228-1240.

Chen, X., And Z. Liao (2014): "Sieve M inference on irregular parameters," Journal of Econometrics, 182, 70-86.

Chen, X., Z. Liao, and Y. Sun (2014): "Sieve Inference on Possibly Misspecified Semi-nonparametric Time Series Models," Journal of Econometrics, 178, 639-658.

Chen, X., And D. Pouzo (2009): "Efficient Estimation of Semiparametric Conditional Moment Models with Possibly Nonsmooth Residuals," Journal of Econometrics, 152, 46-60.

Gallant, A. R., and D. W. Nychka (1987): "Semi-nonparametric Maximum Likelihood Estimation," Econometrica, 55, 363-390.

Gentry, M., And T. Li (2014): "Identification in Auctions with Selective Entry," Econometrica, 82, 314-344.

Genz, A. (1992): "Numerical Computation of Multivariate Normal Probabilities," Journal of Computational and Graphical Statistics, 1(2), 141-149.

Gillen, B. (2010): "Identification and Estimation of Level- $k$ Auctions," Discussion paper, Caltech.

Gilley, O. W., and G. V. Karels (1981): "The Competitive Effect in Bonus Bidding: New Evidence," Bell Journal of Economics, 12, 637-648.

Guerre, E., I. M. Perrigne, and Q. Vuong (2000): "Optimal Nonparametric Estimation of First-Price Auctions," Econometrica, 68, 525-574.

(2009): "Nonparametic Identification of Risk Aversion in First-Price Auctions Under Exclusion Restrictions," Econometrica, 77, 1193-1227.

Haile, P. A., K. Hendricks, and R. H. Porter (2010): "Recent U.S. Offshore Oil and Gase Lease Bidding: A Progress Report," International Journal of Industrial Organization, 28, 390-396.

Haile, P. A., H. Hong, and M. Shum (2003): "Nonparametric Tests for Common Values in First-Price Sealed-Bid Auctions," Cowles Foundation Discussion Paper 1444. 
Haile, P. A., And Y. Kitamura (2018): "Unobserved Heterogeneity in Auctions," Discussion paper, Yale University.

Hendricks, K., J. Pinkse, and R. H. Porter (2003): "Empirical Implications of Equilibrium Bidding in First-Price, Symmetric, Common Value Auctions," Review of Economic Studies, 70, 115-145.

Hendricks, K., And R. H. Porter (1988): "An Empirical Study of an Auction with Asymmetric Information," American Economic Review, 78, 865-883.

Review, Papers and Proceedings, 82, 506-511.

Hendricks, K., R. H. Porter, and B. Boudreau (1987): "Information, Returns, and Bidding Behavior in OCS Auctions: 1954-1969," Journal of Industrial Economics, 35, 517-42.

Hendricks, K., R. H. Porter, and R. H. Spady (1989): "Random Reservation Prices and Bidding Behavior in OCS Drainage Auctions," The Journal of Law and Economics, 32, S83-S106.

Hendricks, K., R. H. Porter, and G. TAn (2008): "Bidding Rings and the Winner's Curse," RAND Journal of Economics, 39, 1018-1041.

Hendricks, K., R. H. Porter, and C. A. Wilson (1994): "Auctions for Oil and Gas Leases with an Informed Bidder and a Random Reservation Price," Econometrica, 62, 1415-44.

Hill, J. B., And A. Schneyerov (2014): "Are There Common Values in FirstPrice Auctions? A Tail-Index Nonparametric Test," Journal of Econometrics, 174, $144-164$.

Hong, H., And M. Shum (2002): "Increasing Competition and the Winner's Curse: Evidence from Procurement," Review of Economic Studies, 69, 871-898.

Hortaçsu, A., AND J. Kastl (2012): "Valuing Dealers' Informational Advantage: A Study of Canadian Treasury Auctions," Econometrica, 80, 2511-2542.

Hu, Y., D. McAdams, and M. Shum (2013): "Identification of First-Price Auctions with Non-Separable Unobserved Heterogeneity," Journal of Econometrics, 174, 186-193.

Hubbard, T. P., H. J. PaArsch, and T. Li (2012): "Semiparametric Estimation in Models of First-Price Sealed-Bid Auctions," Journal of Econometrics, 168, 4-16.

Kitamura, Y., And L. LaAge (2017): "Nonparametric Analysis of Finite Mixtures," Discussion paper, Yale University. 
Komarova, T. (2017): "Extremum Sieve Estimation in $k$-out-of-n Systems," Communications in Statistics: Theory and Methods, 46, 4915-4931.

Kong, Y. (2017a): "Selective Entry in Auctions: Estimation and Evidence," Discussion paper, Rice University.

— (2017b): "Sequential Auctions with Synergy and Affiliation Across Auctions," Discussion paper, Rice University.

Krasnokutskaya, E. (2011): "Identification and Estimation in Procurement Auctions under Unobserved Auction Heterogeneity," Review of Economic Studies, 28, $293-327$.

Krasnokutskaya, E., And K. Seim (2011): "Bid Preference Programs and Participation in Highway Procurement Auctions," American Economic Review, 101, 2653-2686.

LAfFont, J.-J. (1997): "Game Theory and Empirical Economics: The Case of Auction Data," European Economic Review, 41, 1-35.

LAfFont, J.-J., And Q. VuOng (1993): "Structural Econometric Analysis of Descending Auctions," European Economic Review, 37, 329-341.

LAHIRI, S. (2003): Resampling Methods for Dependent Data. Springer-Verlag, New York.

Levin, D., And J. L. Smith (1994): "Equilibrium in Auctions with Entry," American Economic Review, 84, 585-599.

Li, T., I. Perrigne, And Q. Vuong (2000): "Conditionally Independent Private Information in OCS Wildcat Auctions," Journal of Econometrics, 98, 129-161.

- (2002): "Structural Estimation of the Affiliated Private Value Auction Model," RAND Journal of Economics, 33, 171-193.

Li, T., And X. Zheng (2009): "Entry and Competition Effects in First-Price Auctions: Theory and Evidence from Procurement Auctions," Review of Economic Studies, 76, 1397-1429.

Ma, S., And M. Kosorok (2005): "Robust Semiparametric M-Estimation and the Weighted Bootstrap," Journal of Multivariate Analysis, 96, 190-217.

Marmer, V., A. Schneyerov, and P. Xu (2013): "What Model for Entry in First-Price Auctions? A Nonparametric Approach," Journal of Econometrics, 176, $46-58$.

Milgrom, P. R. (1987): "Auction Theory," in Advances in Economic Theory: Fifth World Congress, ed. by T. F. Bewley. Cambridge University Press. 
Milgrom, P. R., And R. J. Weber (1982): "A Theory of Auctions and Competitive Bidding," Econometrica, 50, 1089-1122.

PAARsch, H. J. (1992): "Deciding between the Common and Private Value Paradigms in Empirical Models of Auctions," Journal of Econometrics, 51, 191215.

Pinkse, J., And G. TAN (2005): "The Affiliation Effect in First-Price Auctions," Econometrica, 73, 263-277.

Roberts, J. W. (2013): "Unobserved Heterogeneity and Reserve Prices in Auctions," RAND Journal of Economics, pp. 712-732.

Somaini, P. (2015): "Identification of Auction Models with Interdependent Costs," Discussion paper, Stanford University.

Syrgkanis, V., E. Tamer, and J. Ziani (2018): "Inference on Auctions with Weak Assumptions on Information," Discussion paper, Harvard University.

VAN DER VAART, A., AND J. Wellner (1996): Weak Convergence and Empirical Processes: With Applications to Statistics. Springer-Verlag, New York.

White, H., And J. Wooldridge (1991): "Some Results on Sieve Estimation with Dependent Observations," in Non-parametric and Semi-parametric Methods in Econometrics and Statistics, ed. by W. Barnett, J. Powell, and G. Tauchen, pp. 459-493. Cambridge University Press.

YAO, Q. (2003): "Exponential Inequalities for Spatial Processes and Uniform Convergence Rates for Density Estimation," in Development of Modern Statistics and Related Topics: in celebration of Prof. Yaoting Zhang's 70th birthday, ed. by H. Zhang, and J. Huang, pp. 118-128. World Scientific. 